\title{
The many faces of Pluripotency: in vitro adaptations of a continuum of in vivo states
}

\author{
Sophie Morgani ${ }^{1,2}$, Jennifer Nichols ${ }^{2}$ and Anna-Katerina Hadjantonakis ${ }^{1 *}$ (D)
}

\begin{abstract}
Pluripotency defines the propensity of a cell to differentiate into, and generate, all somatic, as well as germ cells. The epiblast of the early mammalian embryo is the founder population of all germ layer derivatives and thus represents the bona fide in vivo pluripotent cell population. The so-called pluripotent state spans several days of development and is lost during gastrulation as epiblast cells make fate decisions towards a mesoderm, endoderm or ectoderm identity. It is now widely recognized that the features of the pluripotent population evolve as development proceeds from the pre- to post-implantation period, marked by distinct transcriptional and epigenetic signatures. During this period of time epiblast cells mature through a continuum of pluripotent states with unique properties. Aspects of this pluripotent continuum can be captured in vitro in the form of stable pluripotent stem cell types. In this review we discuss the continuum of pluripotency existing within the mammalian embryo, using the mouse as a model, and the cognate stem cell types that can be derived and propagated in vitro. Furthermore, we speculate on embryonic stage-specific characteristics that could be utilized to identify novel, developmentally relevant, pluripotent states.
\end{abstract}

Keywords: Pluripotency, Naïve, Intermediate, Formative, Primed, Embryonic stem cells, Epiblast-like cells, Epiblast stem cells, Ground state, Chimaera

\section{Background}

Pluripotency is the potential of a single cell to generate all somatic lineages of the adult organism, comprising mesoderm, endoderm and ectoderm derivatives, as well as the germ cells. During early mammalian development, cells within the epiblast (Epi) of the embryo are pluripotent and go on to form the embryo-proper. As development progresses, a combination of Fibroblast Growth Factor (FGF), Bone Morphogenetic Protein (BMP), Wnt and Nodal signaling triggers the loss of pluripotency by driving differentiation of the Epi into specialized, developmentally-restricted fates [1]. In the mouse, pluripotent cells are present from embryonic day (E) 3.5 to 8.0, representing approximately one quarter of the gestation period (Fig. 1). During this time, the pluripotent population evolves, characterized by

\footnotetext{
*Correspondence: hadj@mskcc.org

${ }^{1}$ Developmental Biology Program, Sloan Kettering Institute, Memorial Sloan Kettering Cancer Center, New York, NY 10065, USA

Full list of author information is available at the end of the article
}

changes in gene expression, epigenetic profile and functional properties. While distinct "naïve" and "primed" pluripotent states have been described, that correspond to the pre and post-implantation Epi respectively [2], the progressive nature of development means that a broad continuum of pluripotency likely exists within the developing embryo (Table 1). To define additional intermediate states, a high-resolution gene expression map of these embryonic stages is required, a task made difficult by the rapid advancement of in vivo development and limited availability of material.

It is, however, possible to study pluripotency in a more stable state in vitro using cell lines derived from the embryo [3-6]. These cell lines offer a tool to study early development, as well as a reservoir of unspecified cells with significant therapeutic potential. Pluripotent stem cell (PSC) lines can self-renew indefinitely while maintaining the capacity to differentiate into all cell types in vitro [7-9] and in vivo [4, 10-12]. Many iterations of pluripotency can 


\section{Pluripotent cells \\ Pluripotent-containing region \\ Extraembryonic: TE-derived \\ Extraembryonic: PrE-derived \\ PS and derivatives \\ Anterior NE}
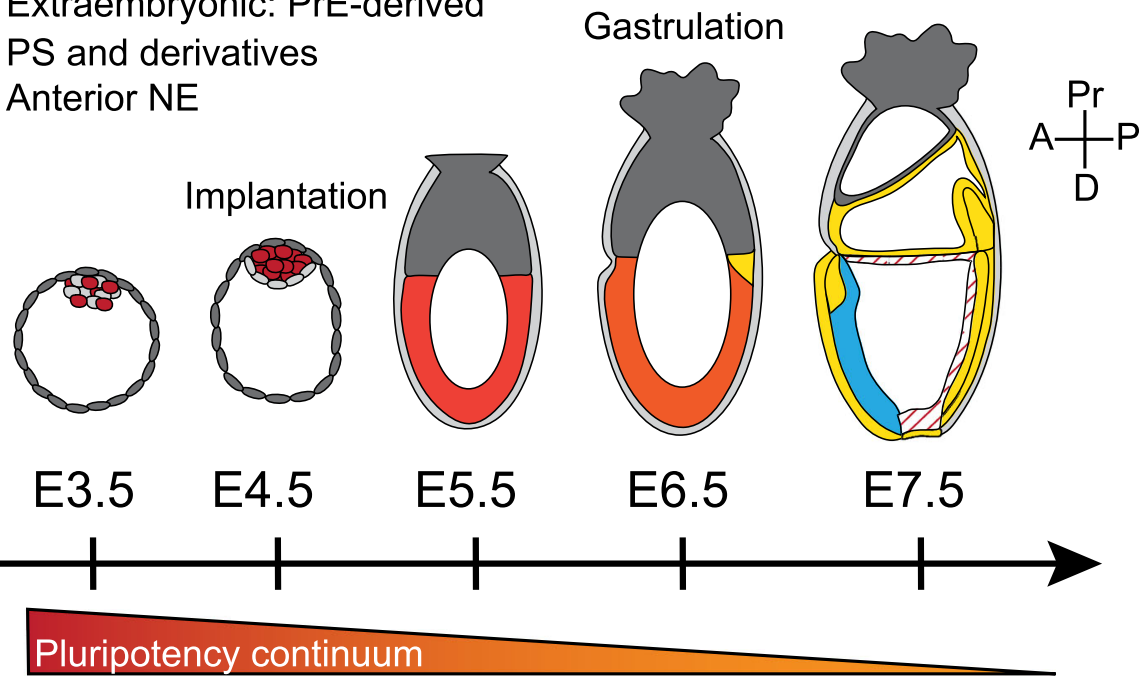

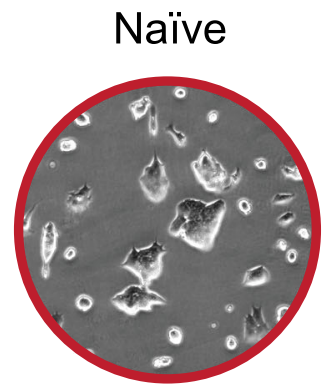

ESCs

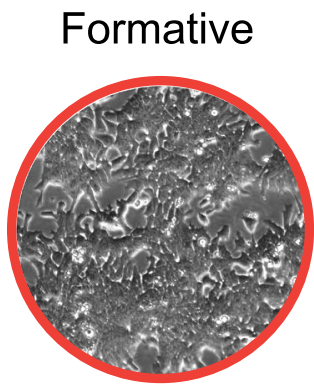

EpiLCs

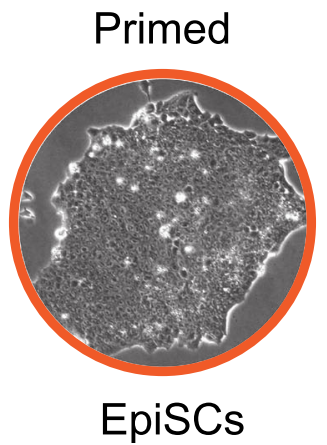

Fig. 1 A schematic diagram depicting the relationship between in vitro and in vivo pluripotent state progression. The diagram depicts the location of pluripotent cells (red) within the developing mouse embryo from embryonic day (E) 3.5 to E7.5. Prior to E3.5, cells of the pre-implantation embryo are 'totipotent', capable of generating both embryonic and extraembryonic cell types. At E3.5, cells in the ICM of the blastocyst are a heterogeneous mix of epiblast (Epi) and primitive endoderm (PrE) precursors. Epi cells are pluripotent and will generate all cells of the embryo-proper, including the germ cells, proper while PrE cells will generate extraembryonic cell types such as the yolk sac. The outer trophectoderm (TE) cells will generate extraembryonic cell types including the fetal portion of the placenta. One day later, at E4.5, the Epi and PrE cells are specified and become physically segregated into two distinct layers and the embryo implants into the uterus. At early post-implantation stages (E5.5) the Epi is in an entirely undifferentiated pluripotent state. At E6.5, cells within the proximal posterior of the embryo are exposed to differentiation-promoting signals from both embryonic and extraembryonic lineages that stimulate the onset of gastrulation and differentiation of cells as they enter the primitive streak (PS) region (yellow). By E7.5, the PS has extended distally and PS derivatives including extraembryonic mesoderm, embryonic mesoderm and definitive endoderm are being generated. The anterior Epi has also started to differentiate into anterior neurectoderm (NE). Pluripotency is lost at approximately E8.0. Pluripotent stem cell lines can be maintained in vitro and appear to resemble various embryonic stages of pluripotency. While embryonic stem cells (ESCs) can be derived from embryos from E3.5 and E7.5 and epiblast stem cells (EpiSCs) can be derived from embryos between E3.5 and E8.0, ESCs resemble the naïve pluripotent state (blue) existing in the early pre-implantation embryo while EpiSCs resemble primed pluripotent cells (green) of the late post-implantation Epi during gastrulation. Intermediate or formative states of pluripotency (orange), between the naïve and primed states likely exist in the embryo. While a number of potential states have been isolated, Epi-like cells (EpiLCs), generated from ESCs in vitro, have been most clearly defined in relation to the embryo and are more transcriptionally similar to E5.75 Epi than EpiSCs are. Representative brightfield images of ESC, EpiLC and EpiSC cultures are shown. Extraembryonic lineages are depicted in gray; dark gray lineages are TE-derived and light gray lineages PrE-derived. $\mathrm{A}=$ anterior, $\mathrm{P}=$ posterior, $\mathrm{Pr}=\operatorname{proximal,} \mathrm{D}=$ distal

be propagated in culture depending on the conditions utilized (Table 2). It is currently unclear whether these represent distinct points on a pluripotency spectrum, which also arise in vivo during normal development, or if they are merely culture artifacts. Here, we discuss our current knowledge of PSC states, focusing on the mouse model, in which most research has been carried out. 
Table 1 Overview of pluripotent states and defining characteristics. Although naïve and primed states of pluripotency have been well characterized, there is no clear consensus of the expected characteristics of their intermediate pluripotent states. This table highlights a number of defining characteristics of naive and primed pluripotent states, and stipulates on the characteristics that intermediate states might encompass. Although a spectrum of intermediate states may exist, here we hypothetically distinguish between two potential intermediate states, 'Intermediate 1', the epiblast immediately after implantation and 'Intermediate 2' the epiblast at the onset of gastrulation

\begin{tabular}{|c|c|c|c|c|}
\hline $\begin{array}{l}\text { Pluripotent } \\
\text { state }\end{array}$ & $\begin{array}{l}\text { Corresponding } \\
\text { embryonic stage }\end{array}$ & Gene expression & Epigenetic profile & Functional potential \\
\hline Naïve - ESCS & E3.5-4.5 & $\begin{array}{l}\text { Oct4, Sox2, Nanog, KIf4, Stella, Rex1, Gbx2, } \\
\text { Tbx3, Pecam, SSEA-1, Alkaline phosphatase }\end{array}$ & $\begin{array}{l}\text { X reactivation. DE-controlled Oct4 } \\
\text { expression. }\end{array}$ & $\begin{array}{l}\text { Pre-imp. Chimaeras. } \\
\text { Poor PGCLC generation. }\end{array}$ \\
\hline Primed - EpiSCs & E7.25-8.0 & $\begin{array}{l}\text { Oct4, Sox2, Nanog, SSEA-1, Fgf5, Oct6, Otx2, } \\
\text { Brachyury, FoxA2, Sox17, Gata4, Gata6 }\end{array}$ & $\begin{array}{l}\mathrm{X} \text { inactivation. PE-controlled Oct4 } \\
\text { expression. }\end{array}$ & $\begin{array}{l}\text { Post-imp. Chimaeras. } \\
\text { Poor PGCLC generation. }\end{array}$ \\
\hline Intermediate 1 & E5.0-6.25 & $\begin{array}{l}\text { Oct4, Sox2, low Nanog, SSEA-1, Fgf5, Oct6, } \\
\text { Otx2 (no PS or lineage markers) }\end{array}$ & $\begin{array}{l}X \text { reactivation, Equal Oct4 } \\
\text { regulation by } D E \text { and } P E \text { ? }\end{array}$ & $\begin{array}{l}\text { Pre and post-imp. } \\
\text { Chimaeras. } \\
\text { Efficient PGCLC generation. }\end{array}$ \\
\hline Intermediate 2 & E6.25-7.25 & $\begin{array}{l}\text { Early PS/mesoderm markers e.g. } \\
\text { Nanog, Brachyury. } \\
\text { No endoderm markers. }\end{array}$ & $\mathrm{X}$ inactivation. & $\begin{array}{l}\text { Post-imp. Chimaeras. } \\
\text { Reduced PGCLC } \\
\text { generation. }\end{array}$ \\
\hline
\end{tabular}

ESCs embryonic stem cells, EpiSCs epiblast stem cells, $E$ embryonic day, $D E$ distal enhancer, $P E$ proximal enhancer, pre-imp. pre-implantation, post-imp. post-implantation, PGCLC primordial germ cell-like cell, PS primitive streak

\section{Embryonic stem cells}

(i) derivation and culture conditions

Mouse embryonic stem cells (ESCs) were the first PSC lines to be derived from developing embryos. ESCs are routinely derived from and resemble the naïve Epi of E3.5-4.5 pre-implantation embryos [4, 12-14]. While ESCs can also be derived from embryos as early as E0.5 $[6,13,15]$, these embryos develop ex vivo to a late blastocyst stage before ESCs emerge [13]. Conversely, attempts to derive PSC lines from later stage embryos using naïve ESC culture conditions have been largely unsuccessful, consistent with the distinct nature of the pre and post-implantation Epi. Intriguingly, while ESC lines cannot be established from whole explants of postimplantation Epi $[3,16,17]$, they can be derived from dissociated E7.5 Epi [18, 19] implying that a refractory niche may be present within the intact tissue. These cells, referred to as reprogrammed Epi ESC-like cells (rESCs), undergo transcriptional and epigenetic changes consistent with reprogramming to an earlier developmental state during the derivation procedure [18]. In vitro equivalents of the post-implantation Epi (epiblast stem cells, see below) can also occasionally revert to an ESC state [18-23], a process enhanced by genetic manipulation [16, 24-26]. Pluripotent cells therefore maintain a degree of plasticity and can deviate from, or even reverse, the normal developmental trajectory if a permissive environment is provided.

ESCs were first derived in poorly defined serum containing medium and were maintained on a bed of mitotically inactivated fibroblasts (so-called 'feeder cells') $[4,12]$. The critical factors provided by each of these components are now known to be activators of the leukemia inhibitory factor (LIF) [27, 28] and BMP [29] pathways respectively (Fig. 2, Table 2). While ESCs can be maintained in defined conditions with LIF and BMP4 [29-31] (or other interleukin-6 family members [32-36]), serum and LIF (SL) is favored as an economical alternative with enhanced plating efficiency [29]. LIF and BMP stimulate the expression of pluripotencyassociated genes in ESC cultures [29, 31, 37], and BMP may additionally inhibit differentiation-inducing Mitogen-activated protein kinase/Extracellular signalregulated kinase (MAPK/ERK) signals [38, 39] (Fig. 2). BMP also maintains Epi pluripotency in vivo by preventing premature neural specification [40] and, while LIF is not required for normal pre-implantation development [41-45], it maintains self-renewal during diapause [43]. Therefore, ESCs retain the key signaling properties of their embryonic cell of origin and may be similar to the Epi of diapause embryos.

\section{(ii) transcriptional and epigenetic profiles}

Although ESCs can be derived from multiple developmental stages, they retain no clear 'memory' of their developmental origin and converge at a transcriptional and epigenetic state similar to the Epi of the E3.5-4.5 blastocyst [13, 46]. ESCs exhibit an open chromatin structure and high levels of global transcriptional activity, similar to the pre-implantation embryo, that become more restricted as differentiation proceeds [47-50]. This active chromatin state is characterized by large regions of DNA hypomethylation, histone acetylation and H2K4me3 [51, 52] and is attributed in part to factors recruited to the citrullination modification on histone $\mathrm{H} 1$ [53, 54]. Furthermore, female ESC lines exhibit X chromosome inactivation, an epigenetic hallmark of the naive pluripotent state present at this time in vivo [55], although the level of $\mathrm{X}$ chromosome methylation varies between individual cells [56]. ESCs also express a cohort 


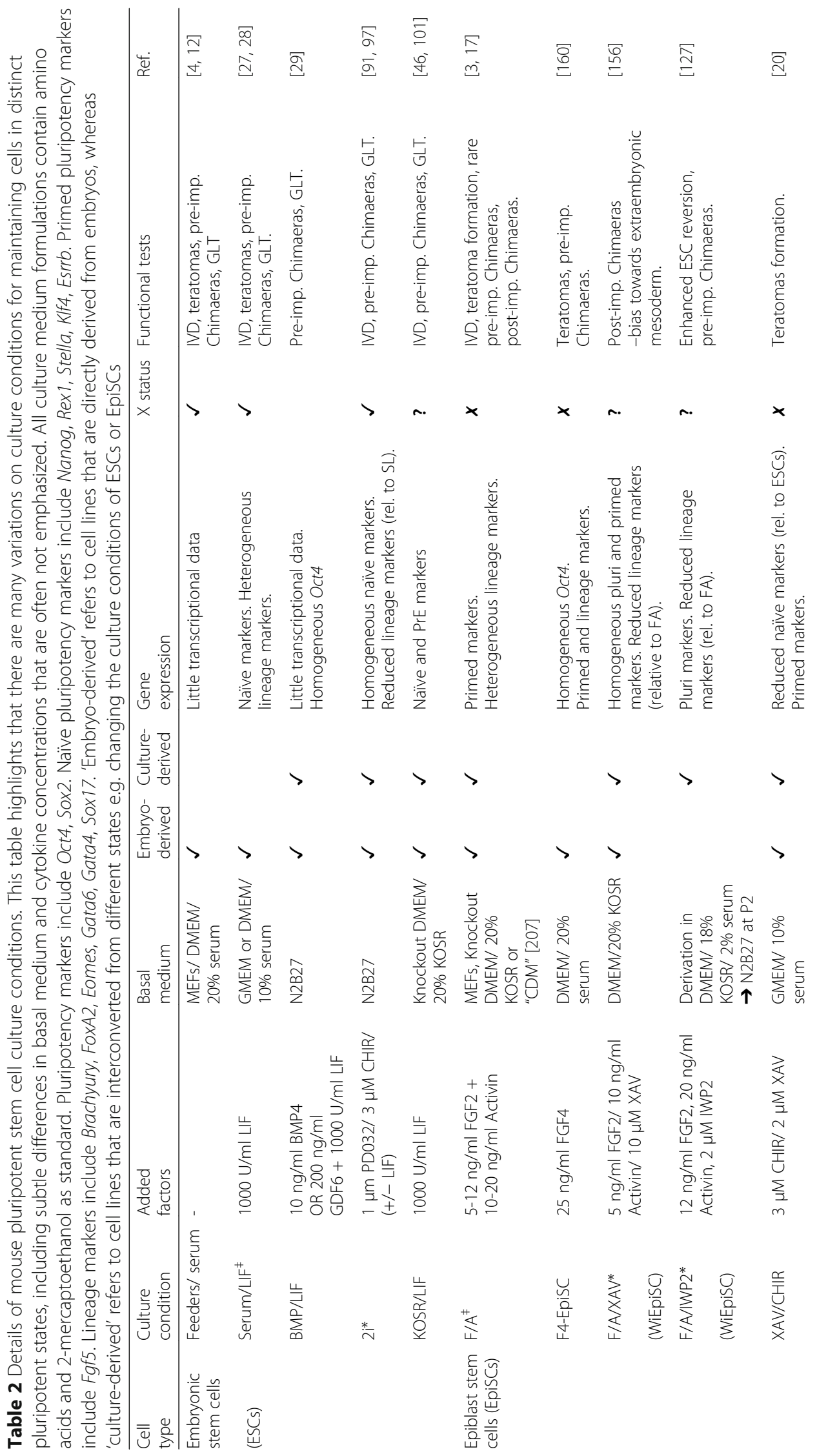


Morgani et al. BMC Developmental Biology (2017) 17:7

Page 5 of 20

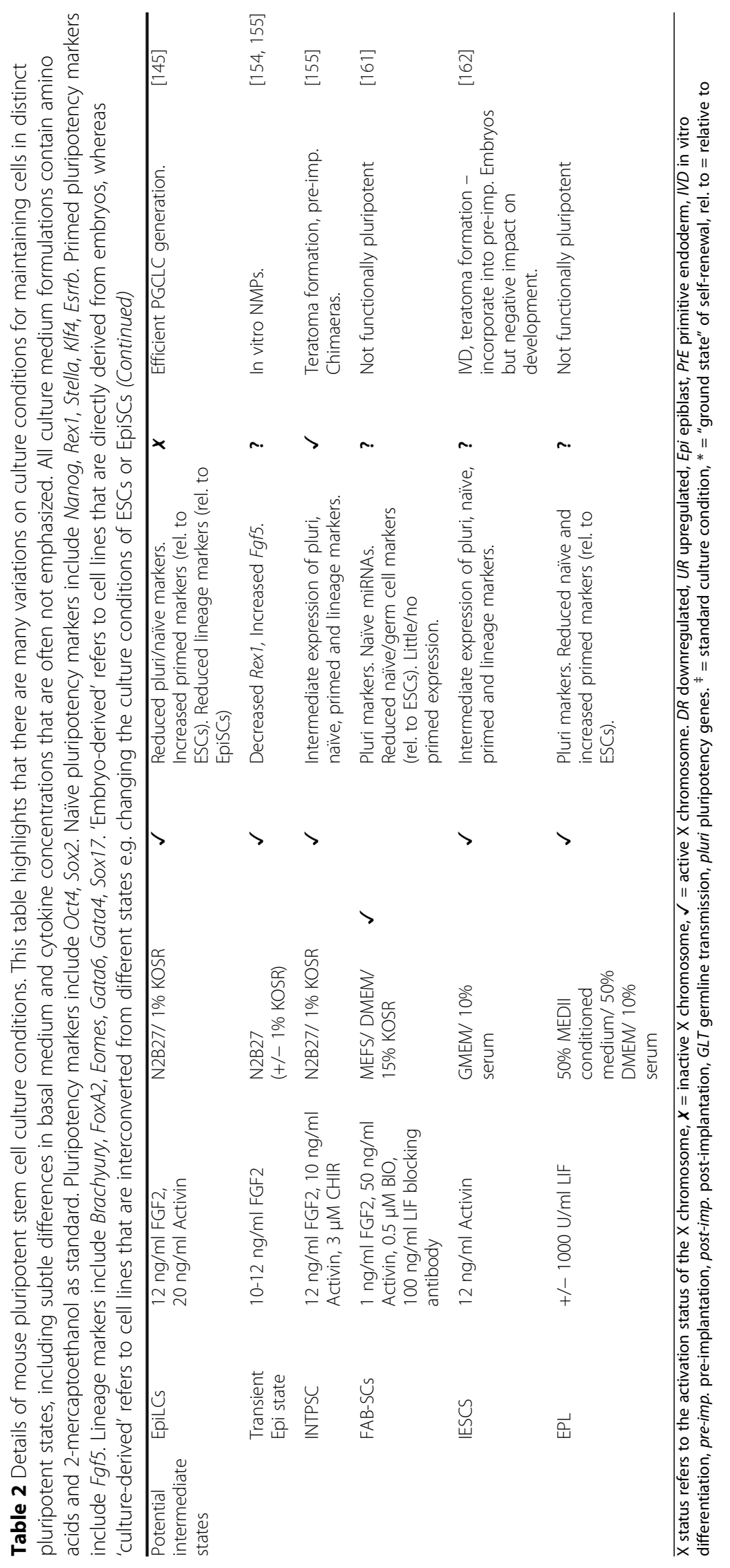




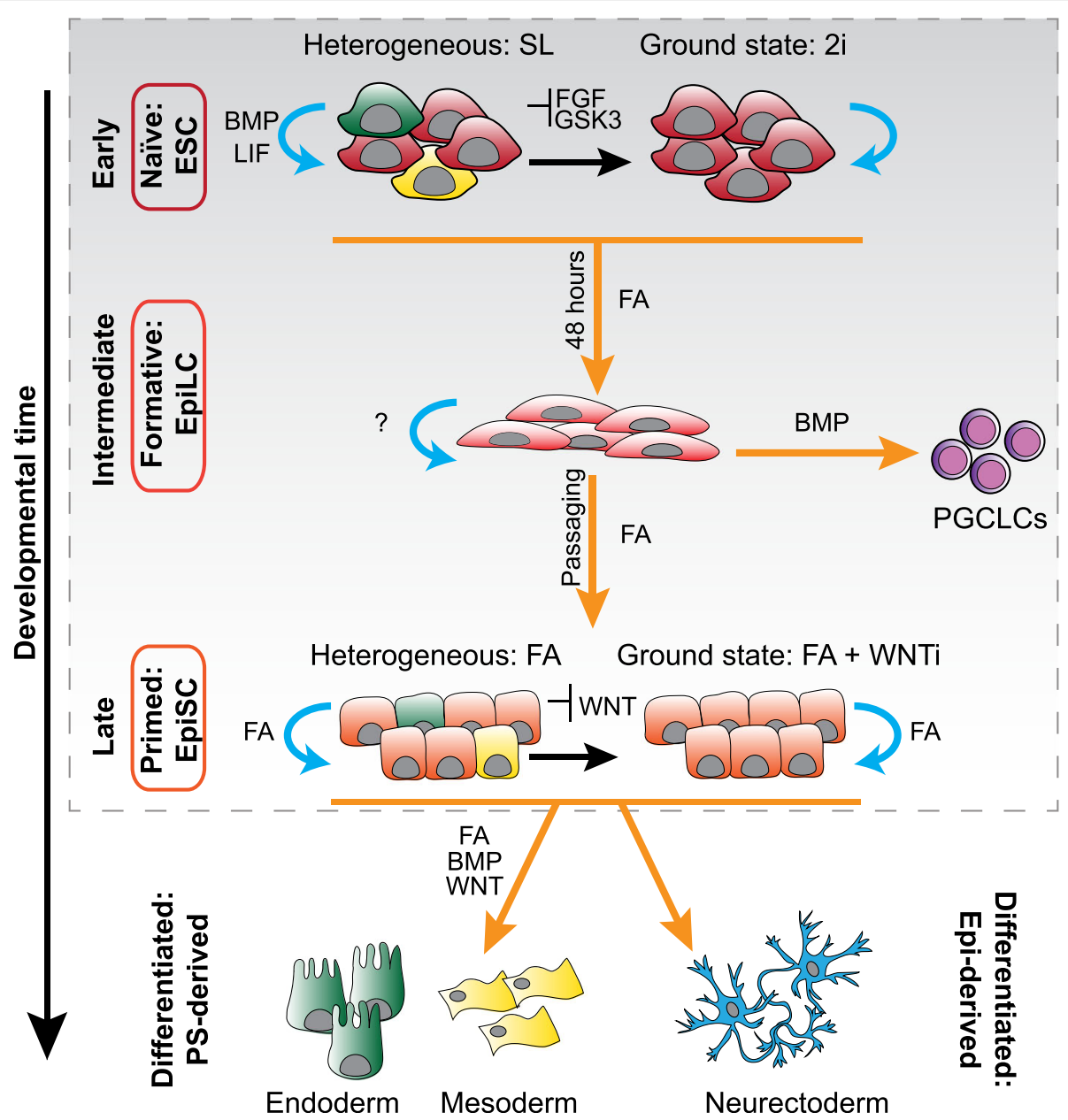

Fig. 2 The role of signaling pathways in self-renewal and differentiation of in vitro pluripotent stem cell populations. Embryonic stem cells (ESCs) represent a naïve state of pluripotency similar to the pre-implantation epiblast (Epi). ESCs are routinely maintained in a self-renewing state in serum (a source of BMP) and LIF (SL). Under these conditions, ESC cultures are heterogeneous and contain subpopulations of lineage-primed cells (yellow and green cells) i.e. cells that coexpress germ layer markers alongside pluripotency markers, and are biased in differentiation towards particular lineages. A more homogeneous ESC state can be generated by blocking FGF signaling using a MEK inhibitor (PD0325901), and activating Wnt signaling using a GSK3 inhibitor (CHIR99021), a condition known as ' $2 i$ '. In these conditions, self-renewal occurs in the absence of external signals, although cell propagation is enhanced in the presence of Wnt pathway activity through CHIR99021. This state is referred to as the naïve or "ground state" of pluripotency. ESCs can be pushed further along the differentiation trajectory by culturing in the presence of FGF and Activin (FA) for 48 hours to generate a cell state referred to as Epi-like cells (EpiLCs). This is a transient cell state, and it is unknown whether self-renewing EpiLCs can be captured by the addition of other factors. It is also not known whether EpiLCs are a homogeneous population of cells. Upon further differentiation in FA over multiple passages, cells resemble a primed state of pluripotency akin to the later post-implantation Epi, referred to as epiblast stem cells (EpiSCs). While FA promotes differentiation of ESCs and EpiLCs, it promotes EpiSC self-renewal. EpiSCs can be derived from ESCS in culture or directly from embryos with FA. When grown in FA, EpiSCs, like ESCS in SL, are heterogeneous and contain lineage-primed populations. While activation of Wnt signaling promotes a naïve ground state of self-renewal, inhibition of Wnt signaling promotes a more homogeneous primed ground state of self-renewal. Therefore cells in naïve and primed pluripotent states respond to signaling factors with opposite outcomes, Wnt and BMP promote self-renewal of the naive state but differentiation of the primed state of pluripotency and conversely FA promote differentiation of the naïve state but self-renewal of the primed state of pluripotency. Addition of BMP4 and WNT3A in combination with FA stimulates further differentiation of EpiSCs into PS-derived mesoderm and endoderm, while in the absence of FA, BMP or Wnt EpiSCs differentiate to neurectoderm [127]. EpiLCs are the only pluripotent state that has been shown to efficiently generate primordial germ cell-like cells (PGCLCs). Presumably, ESCs have not yet acquired this capacity, while EpiSCs have lost it. Cells within the dashed box are within the pluripotency spectrum while cells outside have differentiated. Blue arrows indicate self-renewal. Orange arrows denote the direction of differentiation along the developmental trajectory

of transcription factors characteristic of the preimplantation Epi including Oct4 (Pou5f1), Sox2, Nanog, Klf4, Stella (Dppa3) and Rex1 (Zfp42) [57, 58] (Fig. 3).
Furthermore, as in the blastocyst, Oct4 expression is regulated by its distal enhancer element [59]. Some of the key targets of this transcription factor network include 

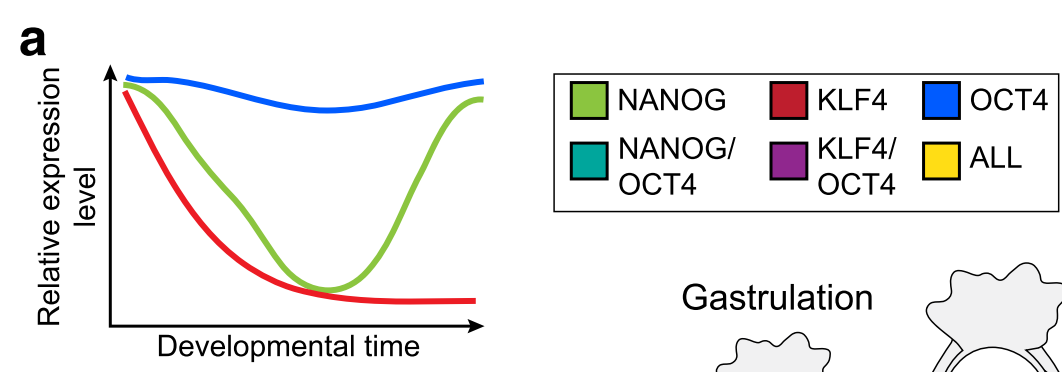

b
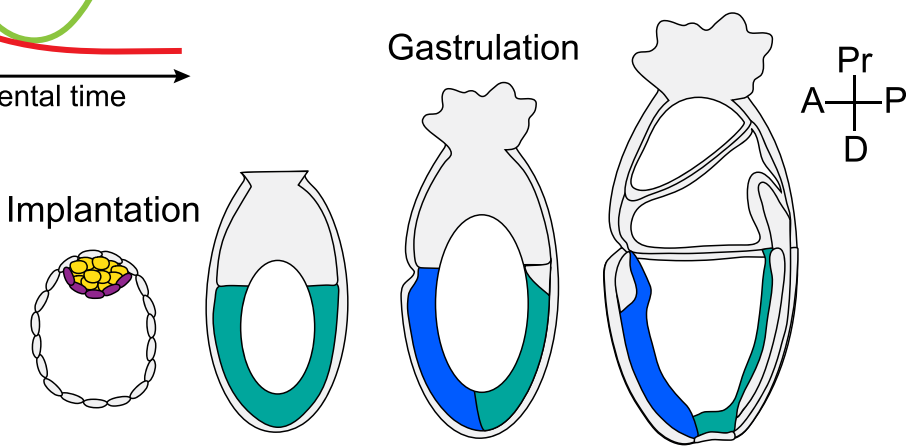

\section{$\begin{array}{lllll}\text { E3.5 } & \text { E4.5 } & \text { E5.5 } & \text { E6.5 } & \text { E7.5 }\end{array}$}

C
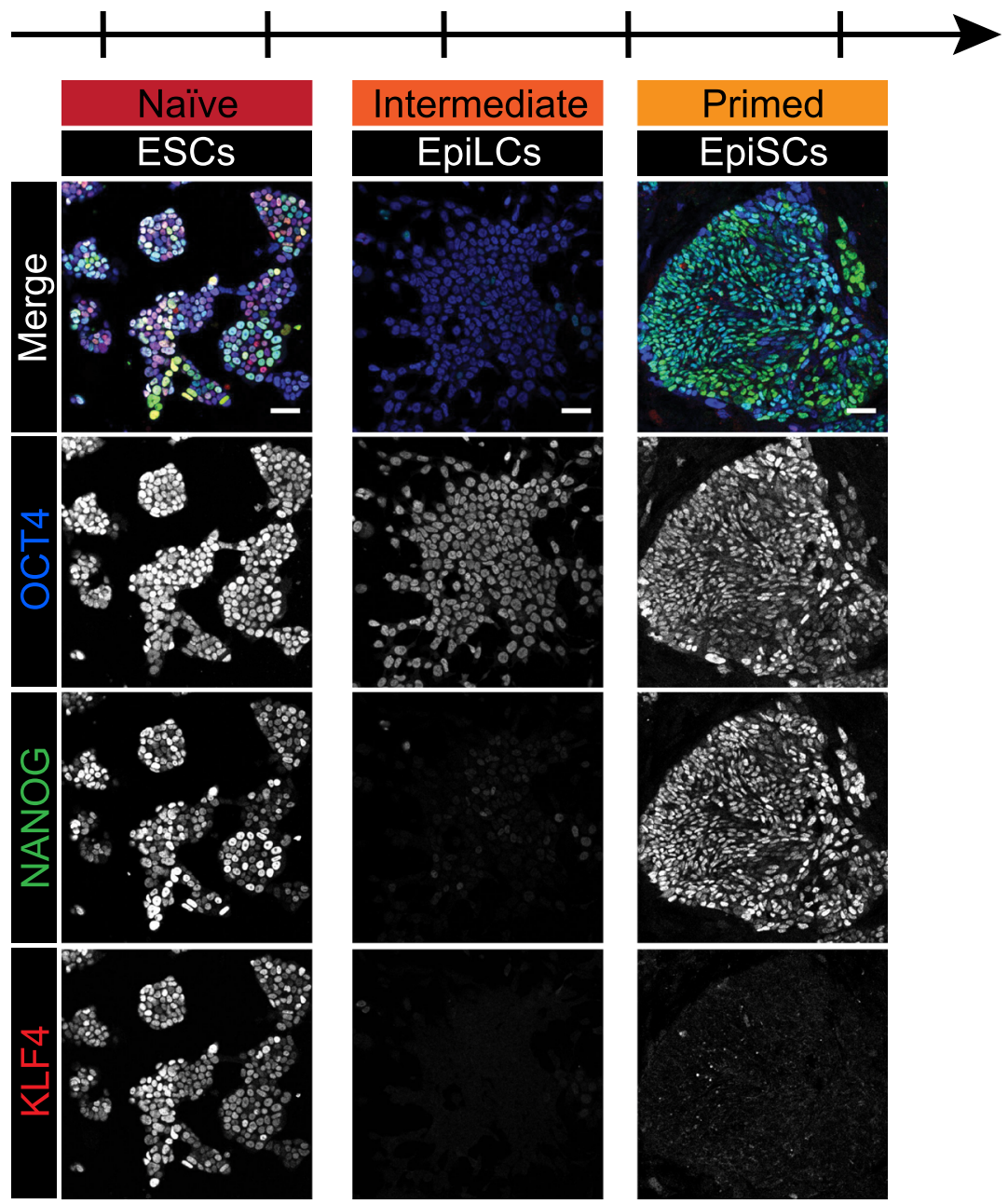

Fig. 3 (See legend on next page.) 


\begin{abstract}
(See figure on previous page.)
Fig. 3 Different pluripotent states have distinct expression profiles. a. Schematic diagram illustrating the change in relative protein expression levels of the pluripotency-associated genes, NANOG, KLF4 and OCT4 during the transition from a naïve to a primed state of pluripotency. KLF4 is lost as cells exit the naïve state of pluripotency, NANOG is transiently downregulated and OCT4 is maintained at similar levels throughout this period. $\mathbf{b}$. Schematic diagram showing the expression domains of NANOG, KLF4 and OCT4 from embryonic day (E) 3.5 to 7.5 of development. NANOG, KLF4 and OCT4 are all expressed within the ICM of the early blastocyst. While OCT4 is relatively homogeneous, KLF4 and NANOG are both heterogeneously expressed. At E4.5, the epiblast (Epi) homogeneously expresses all 3 of these markers, while the primitive endoderm expresses low levels of OCT4 and KLF4 but not NANOG. At early implantation (E5.5), KLF4 expression is lost and OCT4 and NANOG are coexpressed throughout the Epi. By E6.5-7.5, OCT4 continues to be expressed throughout the Epi while NANOG is restricted to the posterior Epi. c. Representative confocal optical sections of ESC, EpiLC and EpiSC cultures. All cell lines were derived from the 129/Ola E14 parental ESC line. ESCs were maintained in serum and LIF and expressed OCT4, NANOG and KLF4 heterogeneously. EpiLCs expressed OCT4, but downregulated NANOG, and lost KLF4 expression. EpiSCs (derived by culture of E14 ESCs in FGF and Activin for $>20$ passages) expressed high levels of NANOG and OCT4, but no KLF4
\end{abstract}

families of micro RNAs (miRNAs) that regulate cell cycle progression in the self-renewing state [60-62]. These core transcription factors and miRNAs maintain self-renewal in vitro and can even induce an ESC-like identity when ectopically expressed in somatic cells [63-66].

However, the pluripotent state in vivo is transient and in a state of constant flux, hence, although ESCs share many similarities with the early embryo, they also employ unique mechanisms to stabilize their state of pluripotency. Genes that regulate pluripotency in vitro are not necessarily required for early Epi development e.g. Esrrb and Tbx3 [67, 68] and the cohorts of bivalent genes, those with both permissive (H3K4me3) and repressive (H3K27me3) epigenetic marks at their promoters, differ between embryos and ESCs [69-71]. Additionally, compared to the pre-implantation embryo, ESCs express high levels of repressive epigenetic factors [72], which may act to shut down the differentiation program. In fact, several thousands of genes alter their expression during ESC derivation [72] and, although the relevance of this is not fully understood, the majority function in growth and metabolism [72], suggesting that the current in vitro culture milieu may not accurately mirror the in vivo environment.

ESCs maintained in standard SL conditions are extremely heterogeneous (Figs. 2 and 3c). Global transcriptional analysis of single cells revealed two classes of heterogeneous gene expression, genes that are expressed bimodally, or those that are expressed in only a small number of cells, but at high levels - referred to as sporadic expression [73]. Subpopulations of cells have been identified that share transcriptional similarities, not only with the pre-implantation Epi [74-76] but also with endoderm [77] and later primed Epi [76, 78]. Furthermore, a subpopulation similar to the 2-cell embryo exists in ESC cultures, which exhibits expanded functional potency and can contribute to both embryonic and extraembryonic lineages in chimaeras [79-81]. Zscan4, specifically expressed within this population, is involved in maintaining telomere length, critical for ESC expansion in vitro [82-84]. It is currently unclear whether these subpopulations are also present in newly established ESC lines or if they emerge gradually in response to extended culture in the in vitro environment. As sampling of this 2-cell state maintains telomere length [82-84], it may arise as a mechanism to limit DNA damage during prolonged culture. Rather than marking stable factions of cells, the transcriptional heterogeneity among ESCs represents a dynamic landscape of interconverting states. In vivo, the blastocyst inner cell mass (ICM) is also a mix of Epi and primitive endoderm (PrE) precursors $[85,86]$ and, although there is no evidence that these populations interconvert during normal development [87], it is unknown whether this would occur if the period of pluripotency were to be prolonged, for example during diapause.

The source of ESC transcriptional heterogeneity has been variously attributed to cell cycle, transcriptional bursting, differences in colony size and density, partial differentiation or a combination of the above. As bivalent genes show a tendency towards heterogeneous expression, epigenetic modifications may also influence transcriptional dynamics $[73,88]$. In reality, ESCs exist in a precarious balancing act of pluripotency and differentiation, prompted by their endogenous production of two factors with opposing functions: LIF, which supports self-renewal, and the differentiation-promoting factor FGF [89]. This signaling tug-of-war could conceivably manifest as cells that are transcriptionally teetering on the edge of different states. The fact that ESC cultures are further supplemented with exogenous LIF means that, although spontaneous differentiation occurs, in the majority of cases self-renewal wins. Consequently, blocking either signal forces cells in one or other direction. Withdrawal of LIF stimulates terminal differentiation while blocking FGF confines cells to a more homogeneous state of self-renewal, referred to as the "ground state" of naïve pluripotency $[2,90]$. This ground state is achieved by culturing ESCs in defined serum-free medium (N2B27) with small molecule inhibitors of the MAPK/ERK pathway (PD0032, a MEK inhibitor) acting downstream of FGF and of Glycogen Synthase Kinase 3 (GSK3) (CHIR99021 or CHIR), together referred to as $2 \mathrm{i}$ 
medium [91] (Table 2). In this context, inhibition of GSK3 stimulates Wnt activity, which relieves TCF3mediated repression of pluripotency-associated genes and prevents differentiation towards a later Epi state [92-94]. LIF is not required in 2i medium but enhances the efficiency of ESC clonal expansion [92]. ESCs cultured in 2i exhibit reduced expression of lineage markers and almost no spontaneous differentiation [95]. Blocking the processing of miRNAs in ESCs can stimulate a similar state of pluripotency [73]. However, single-cell approaches suggest that ESCs in these conditions are not entirely homogeneous as they contain rare cell populations similar to the 2-cell state and extraembryonic lineages [96, 97].

While SL and 2i are most commonly used to maintain ESCs, numerous other systems have been developed that support or enhance self-renewal including additional small molecule inhibitors [98-100], and synthetic serum substitutes, such as knockout serum replacement (KOSR) [101] (Table 2). While ESCs propagated in these distinct conditions all satisfy the functional definition of pluripotency, they have different morphological, transcriptional and epigenetic characteristics. When compared to embryos, SL-cultured ESCs are transcriptionally similar to E4.5-5.5 Epi and extraembryonic lineages [13, 46, 72], 2i-cultured ESCs correlate with earlier E3.5-4.5 ICM or Epi [13, 46, 97] (Fig. 1) and KOSR-cultured ESCs show a surprising transcriptional resemblance to endoderm $[13,46]$. ESCs cultured in $2 \mathrm{i}$ and KOSR also display hypomethylated DNA relative to ESCs in SL $[95,102]$. While these findings could be interpreted as different culture conditions capturing discrete points on the pluripotency spectrum, the majority of data have been acquired from bulk cultures and could instead represent changes in the relative levels of particular subpopulations. Single-cell transcriptomic analyses suggest that SL cultures are, in fact, comprised of two main cell populations, one similar to E2.5-3.5 embryos, and another corresponding to the Epi at approximately E5.5 [103]. It is therefore now understood to be the combination of these states that results in a bulk transcriptional signature similar to E4.5 embryos.

\section{(iii) functional potential}

While transcriptional data provides some insight into the nature of ESC states, functional assays are required to assess pluripotential. When transferred to differentiation-promoting conditions in vitro or reintroduced into host embryos, ESCs can differentiate into derivatives of all germ layers. Traditionally, low-density monolayer differentiation was used as a simple, rapid means of testing functional potential. In serumcontaining medium without LIF, ESCs form endoderm and mesoderm while neural differentiation requires serum-free medium (N2B27) [29, 104]. ESCs have also been reported to generate extraembryonic primitive endoderm (PrE) in vitro $[105,106]$. However, as the majority of markers used to define these cells are also expressed in embryonic definitive endoderm, it is difficult to distinguish between these possibilities. A multitude of directed differentiation protocols have also been established, using cytokine and small molecule inhibitor cocktails to push cells more uniformly in a particular developmental direction. While the result is often a good approximation of the target cell type, on many occasions these differentiated cells are not fully functional. Therefore, a concerted effort is being made to develop 3D differentiation protocols, such as embryoid body (EB)-like structures or organoids, which more accurately mimic the complex in vivo environment $[107,108]$.

The gold standard test of pluripotency is whether cells can successfully incorporate into embryos and resume the normal developmental program. ESCs can incorporate into embryos at a range of pre-implantation stages $[10,46,109-111]$, and contribute most efficiently to Epiderivatives, but also at low levels to extraembryonic lineages $[10,46,80,97,112]$. The frequency of ESC contribution to extraembryonic cell types can be enhanced by selecting for particular subpopulations [77, 97] or by culture in $2 \mathrm{i}$ or KOSR [46, 97], consistent with the notion that ESCs maintained in these conditions correlate to earlier developmental stages than ESCs in SL and, as such, may have a less restricted functional potential. Remarkably, when reintroduced into developmentally compromised tetraploid embryos, live-born mice can be generated entirely from ESCs [113-116]. While the capacity of ESCs to generate primordial germ cell-like cells (PGCLCs) in vitro is limited [117], they can, albeit inefficiently, contribute to the germline in vivo following maturation through a later Epi state. In contrast, when ESCs are introduced into post-implantation embryos, they cannot integrate or differentiate [11] indicating that the naive state of pluripotency is incompatible with the environment of the pluripotent post-implantation Epi.

\section{Epiblast stem cells}

\section{(i) derivation and culture conditions}

While ESCs resemble pre-implantation stages of development, PSCs have also been derived that are similar to the post-implantation Epi. These are referred to as epiblast stem cells (EpiSCs) (Fig. 1). ESCs and EpiSCs are distinct in behavior, morphology, growth factor requirements, transcriptional and epigenetic profiles and functional properties. EpiSCs can be derived from the post-implantation Epi from E5.5 until E8.0 [3, 17, 118], with decreasing efficiency at later embryonic stages [119] correlating with the gradual loss of pluripotency. Although the pre-implantation ICM rapidly 
differentiates in EpiSC medium [3], cell lines can be derived by first expanding the ICM in minimal medium then transferring outgrowths to conventional EpiSC conditions [120]. This primary step likely facilitates the developmental progression of the pre-implantation Epi to a state with later stage signaling requirements. Cells resembling EpiSCs can also be generated in vitro by long-term culture of ESCs in EpiSC conditions $[16,121]$ (Fig. 2). While, in vivo, Epi maturation from a naïve preimplantation to primed post-implantation state occurs over approximately 2 days, in vitro this is a longer, selective process involving extensive cell death and differentiation [122]. Although ESC-derived EpiSCs (ESDEpiSCs) are morphologically and transcriptionally similar to EpiSC lines derived from embryos, their full transcriptional profile or functional potential has not been directly compared.

EpiSCs are routinely maintained with FGF2 and ACTIVIN A (Activin) (FA conditions) $[3,17]$, a TGF- $\beta$ family member with similar signaling properties to NODAL (Fig. 2, Table 2). In vivo, Nodal maintains the expression of the pluripotency markers Oct4 and Nanog and prevents precocious differentiation of the Epi towards neural lineages $[123,124]$. Similarly, in vitro, Activin regulates Nanog expression in EpiSCs [22, 125]. However, as $\mathrm{Nanog}^{-/-}$EpiSC lines can be generated, this is not the primary mechanism by which Activin signaling maintains self-renewal [118]. In EpiSC cultures, FGF, like Activin, blocks neural differentiation and may also prevent rare reversions of EpiSCs to an ESC-like state $[3,17,22]$. It is not clear whether FGF signaling plays a role in self-renewal of the post-implantation Epi in vivo, although it may regulate proliferation [126]. Whereas ESCs can be maintained in a relatively stable state of self-renewal, EpiSCs undergo high levels of spontaneous differentiation [127]. Dissociation of EpiSCs into single cells promotes cell death and differentiation, which can be reduced by using an inhibitor of Rho-associated, coiled-coil containing protein kinase (ROCK, Y-27632) [128] and passaging cells as clusters with gentle enzymatic dissociation or cell scraping. While ESCs are grown on gelatin, they produce endogenous fibronectin which is important for their self-renewal [129]. In contrast, EpiSCs in feeder-free conditions are grown on an exogenous source of fibronectin although there is limited evidence as to whether this influences the EpiSC state [130].

\section{(ii) transcriptional and epigenetic profiles}

Consistent with in vivo development, EpiSCs show little to no expression of the naïve state markers Rex1, Stella, NrOb1, Gbx2, Klf4, Klf2 and Fgf4 [3, 17] (Fig. 3). While expression of the core pluripotency-associated factors, Oct4, Sox2 and Nanog, is maintained, Oct4 expression is regulated mainly via its proximal enhancer element [59] and Nanog expression is reduced relative to ESCs $[16,131-133]$ corresponding to its downregulation upon embryo implantation $[134,135]$. EpiSCs also express the early post-implantation Epi markers Oct6, Fgf5, Otx2 Lefty and Nodal [3, 17]. However, relative to their in vivo post-implantation Epi counterpart, EpiSCs exhibit elevated expression of markers of adhesion (e.g. Tnc, Col1a1, Col6a1), TGF- $\beta$, MAPK and Wnt-associated genes [119], all factors likely affected by the culture conditions. Compared to ESCs, EpiSCs express a distinct cohort of epigenetic regulators and miRNAs, exhibit an increasingly closed chromatin conformation and utilize distinct enhancer elements [133, 136-138]. The distribution of H3K4me1, a mark of enhancers and actively transcribed genes, varies significantly between ESCs and EpiSCs [138, 139]. This histone modification appears to play an active role in establishing the primed state of pluripotency as genetically perturbing the deposition of this mark results significantly enhances the spontaneous conversion of EpiSCs to a naïve ESC state [139]. EpiSCs also show reduced expression of SMARCAD1 relative to ESCs, a protein that is suggested to block H2K9me3mediated heterochromatin formation [54].

As with ESCs, the fact that EpiSCs can be derived from a wide range of embryonic stages raises the question regards what in vivo stage, if any, they represent. The methylation status of specific promoters in EpiSCs is distinct from the in vivo postimplantation Epi [140] and, although EpiSCs were initially thought to correspond to the early postimplantation Epi around E5.0-6.0, they express markers of later, more differentiated cell types arising during gastrulation, e.g. primitive streak (PS) and mesoderm markers Brachyury, Eomes, Gsc, Mixl1 and Fgf8 as well as endoderm markers Sox17, Gata6, Gata4 and FoxA2 [3, 13, 17, 21, 119, 141, 142]. EpiSCs also express imprinted genes monoallelically [143] and female cell lines have an inactive $X$ chromosome, observed in vivo from E6.5 onwards [16-18, 144]. Surprisingly, 2i-cultured ESCs exhibit a stronger correlation than EpiSCs to pre-gastrulation (E5.75) Epi [145] while EpiSCs are actually most transcriptionally similar to E7.25-8.0 embryos in which gastrulation is already underway [119].

In part these findings can be attributed to the extensive spontaneous differentiation of EpiSCs. This is supported by the fact that, in EpiSC cultures, endoderm genes are mostly expressed by differentiated, SSEA-1negative cells [146]. However, subpopulations of cells exist that coexpress the lineage markers BRACHYURY and FOXA2 alongside the pluripotency markers OCT4, SOX2 and NANOG $[142,146]$. Furthermore, the level of 
expression of markers of the mesoderm and endoderm is inversely correlated with the expression of neurectoderm markers such as Sox1 [142], suggesting that there may be at least two subpopulations within EpiSC cultures. Isolated Brachyury-expressing cells show a propensity to differentiate [127], but they can expand and regenerate a mixed culture [142], indicating that this population, while unstable, is not on an irreversible path to differentiation. Interestingly, subpopulations of EpiSCs representative of later lineages, small fractions of EpiSCs also exhibit characteristics of naïve pluripotency. These cells regulate Oct4 expression using its distal enhancer, express lower levels of Brachyury and Fgf5 and high levels of naive pluripotency markers [78, 131, 147]. Although this population is transcriptionally distinct from ESCs [131] it may represent an intermediate state between naïve and primed pluripotency. To add an additional layer of complexity, considerable variability exists between individual EpiSC lines. For example, some EpiSC lines express BRACHYURY in all cells, others have heterogeneous expression, while other lines do not express BRACHYURY at all. The relative composition of individual lines is also surprisingly stable as sub-clones maintained in different laboratories retain these characteristics [119]. The cause of variation between EpiSC lines is unknown and does not correlate with the stage from which these cell lines were derived or whether they are ESC or embryo-derived [119].

\section{(iii) functional potential}

EpiSCs can generate derivatives of all germ layers both in vitro in cultured cells and in embryo grafting experiments. As with ESCs, when differentiated in vitro in serum-containing medium, EpiSCs mostly generate mesoderm and endoderm, while in serum-free medium they tend towards neurectoderm [17]. Removal or prolonged inhibition of FGF or Activin/Nodal signaling also stimulates neural differentiation $[3,17,118]$. While Wnt and BMP signaling maintain ESC self-renewal, these pathways stimulate EpiSC differentiation into a combination of mesoderm and endoderm lineages [127], consistent with their role during gastrulation (Fig. 2). It has also been suggested that BMP promotes extraembryonic endoderm and trophoblast differentiation [3]. However, as discussed above, many markers are shared between extraembryonic PrE and the epiblast-derived definitive endoderm, as well as between mesoderm and trophoblast; hence it is often difficult to distinguish between these fates using a handful of markers. Subpopulations of EpiSCs are biased towards particular lineages e.g. Brachyury positive cells demonstrate an enhanced capacity to form mesoderm [142] and, as EpiSC lines maintain distinct levels of Brachyury, they also vary in their differentiation efficiency towards particular germ layers [119]. The ability of BMP to induce EpiSC differentiation is via downstream activation of the Wnt pathway [127]. BMP and WNT3a also induce Fgf8 and Nodal expression in EpiSCs [127], this is the same combination of factors that cooperate to initiate gastrulation in vivo, suggesting that differentiation in culture is recapitulating in vivo development.

Cells of the E6.0-7.0 Epi do not contribute to embryonic development when heterotopically introduced into blastocysts [148]. In keeping with this, EpiSCs also have little or no capacity to contribute to pre-implantation embryos [3, 17, 131, 149]. In rare cases of contribution, EpiSCs reactivate their X chromosome [149] suggesting that they have been reprogrammed to a naïve state. Although certain EpiSC subpopulations perform better in these assays [131] their contribution is still low. In part this could be due to adhesion-related incompatibility. ESCs, which can readily contribute to pre-implantation development, and EpiSCs are morphologically distinct; ESCs grow as compact, domed colonies while EpiSCs have a flattened morphology. Additionally, ESCs have homogenous and high levels of E-CADHERIN protein at cell-cell junctions, while EpiSCs have patchy, low levels at their interfaces [127]. Overexpression of E-cadherin $(C d h 1)$ in EpiSCs in part rescues their contribution to pre-implantation embryos [149], but their contribution is still extremely limited, indicating that this is not the primary discordancy. Recently, EpiSCs were grafted into post-implantation host embryos, representing a closer stage-match regards their pluripotent state. While dissociated single cells could not incorporate, groups of cells efficiently integrated into the Epi, dispersing from the graft site and upregulated appropriate lineage markers [11]. EpiSCs incorporated most efficiently when introduced into mid or anterior PS, but remained as clumps when introduced into the posterior PS [119]. When Brachyury-positive EpiSCs were grafted into the PS of post-implantation embryos, they preferentially formed axial mesoderm and definitive endoderm [142] while Brachyury negative cells could not successfully incorporate into the embryo $[11,142]$. This is consistent with the notion that Brachyury negative cells are comparable to the in vivo anterior neurectoderm which does not enter the PS. EpiSCs survive when grafted to later E8.5 embryos, when the host Epi is no longer pluripotent, but do not disperse or upregulate appropriate markers [11].

Although EpiSCs can contribute to all germ layers, they have not demonstrated germline transmission (GLT), mostly due to technical obstacles in assessing this capacity. In rare cases where chimaeras were obtained from EpiSC injection into pre-implantation embryos, GLT was not observed [3, 149], and it is not possible to re-introduce post-implantation chimaeras into recipient females for development to term. However, when grafted 
into post-implantation embryos, EpiSCs give rise to cells that express Alkaline Phosphatase, a characteristics of primordial germ cells (PGCs), in the region where germ cells arise [11, 142]. In vitro EpiSCs can generate PGCLCs only with low efficiency [145, 147] consistent with their transcriptional correlation to E7.2-8.0 embryos that have essentially lost their capacity to generate PGCs [150].

\section{In pursuit of intermediate pluripotent states}

As discussed previously, multiple states of pluripotency have been captured in vitro including naïve ESCs, similar to the pre-implantation Epi, and primed EpiSCs, similar to the gastrulating post-implantation Epi. PSCs are a valuable model for studying and mimicking embryo development and a potentially useful tool for therapeutic purposes. However, to successfully differentiate PSCs into functional cell types in vitro, endogenous development will need to be recapitulated in a step-wise manner. Inevitably, in vitro differentiation is an imperfect imitation of in vivo development and therefore unnecessary ex vivo steps, for example the differentiation of naïve ESCs to a mature Epi state, might potentially introduce errors. Thus, it would be beneficial to access a stable intermediate pluripotent cell state, between ESCs and EpiSCs, equivalent to the post-implantation Epi prior to the onset of germ layer differentiation.

This distinct 'formative' state of pluripotency exists in the embryo at E5.5-6.25, when the naïve transcriptional program has been downregulated, but lineage-associated markers are not yet upregulated [151, 152]. Although there seems to be no clear consensus of what characteristics such a state would encompass, a number of conjectures can be made (Table 1). Whereas both the pre and post-implantation Epi express Oct4, the preimplantation Epi employs the DE, while the postimplantation Epi the PE. In vivo this is not a binary switch [131], hence an intermediate pluripotent state may utilize both enhancers. Furthermore, cells of the early E5.0 Epi can contribute to embryonic development when introduced into blastocysts [148], hence we may expect cells in an intermediate state to maintain this capacity, but potentially also to contribute to development when introduced into post-implantation embryos. One clear-cut expectation is that an intermediate pluripotent state would efficiently generate PGCLCs in response to BMP. PGCs are induced in vivo in response to BMP signaling during a very defined time window. ESCs cannot efficiently produce PGCLCs, as BMP maintains naïve pluripotency, and conversely EpiSCs are representative of a developmental stage where PGC competence is already greatly reduced. Next we discuss a number of novel intermediate pluripotent states that have mostly been defined as exhibiting transcriptional profiles somewhere between naïve and primed states.

\section{(i) Epiblast-like cells (EpiLCs)}

ESCs can differentiate to a state resembling EpiSCs by prolonged culture in FA (ESD-EpiSCs, see above). It is therefore tempting to speculate that this maturation recapitulates normal developmental progression bypassing intermediate pluripotent states that exist within the embryo. In support of this hypothesis, characterization of ESCs after 2 days in FA medium identified a transcriptional state similar to the E5.75 Epi [145], just prior to the onset of gastrulation. These culture conditions are almost identical to those for propagating EpiSCs with the addition of $1 \%$ KOSR to reduce cell death and induce a flattened morphology (Table 2). During these 2 first days, cells rapidly proliferate and show little cell death. Thereafter, on day 3 , a wave of cell death is observed [145] corresponding to the initiation of the highly selective ESD-EpiSC program. Although cell death is observed in vivo at this time [153], it is not on a significant scale suggesting that, while the first 2 days of in vitro conversion to EpiSCs may represent a good model of this transition, later time points may not be representative. ESCs also transit through a state similar to the pregastrulation Epi after 2 days with FGF2 alone [154], although these cells have only been assessed using a limited set of markers and may have a compromised proliferative capacity [155] (Table 2). EpiLCs display hallmarks of pluripotency that are intermediate between ESC and EpiSC states; naïve markers, including Stella, Rex1 and Klf4 (Fig. 3) are downregulated while later differentiation-associated markers, such as Brachyury, FoxA2, Sox17, Lefty, Sox1, are not upregulated to the same extent as in EpiSCs and early Epi markers such as Fgf5 and Oct6 are expressed at the same or higher levels than in EpiSCs. Nanog is also transiently downregulated in EpiLCs [145] (Fig. 3), consistent with the fact that it is downregulated at peri-implantation stages and upregulated again in the PS of gastrulating embryos [134]. EpiLCs efficiently generate PGCLCs [145], but their full functional repertoire, including embryo contribution, has not been explored.

(ii) a homogeneous ground state for primed pluripotency Although EpiLCs represent a promising intermediate state between ESCs and EpiSCs, their transient nature means that any differentiation protocol still needs to begin with ESCs, and hence a stable intermediate pluripotent starting population would be highly desirable. ESC and EpiSC culture conditions have been variously adapted in pursuit of such a state. As discussed above, EpiSCs maintained with FA express later germ layer markers, and exhibit high levels of spontaneous 
differentiation. Eliminating this differentiation could push EpiSCs to a more homogeneous earlier Epi state. As with SL-cultured ESCs, endogenous differentiationpromoting factors destabilize the primed state of pluripotency. While FA mediates EpiSC self-renewal, it promotes differentiation when combined with BMP or Wnt signaling activation. EpiSCs express numerous WNT ligands [127], heterogeneously express Axin, a Wnt pathway component and show non-uniform $\beta$ CATENIN localization [142, 156], hence endogenous Wnt activity is a strong candidate for disrupting EpiSC self-renewal. In vivo, FGF, NODAL, BMP and WNT cooperate to initiate an epithelial-to-mesenchymal transition (EMT), and subsequent differentiation of cells in the proximal posterior region of the Epi [1]. Wnt signaling, downstream of BMP is required for mesoderm formation $[157,158]$. Blocking Wnt signaling maintains the Epi in a prolonged state of pluripotency, while activation pushes the entire Epi to adopt a mesoderm fate [156]. Likewise, exposing FA-cultured EpiSCs to BMP4 or WNT3A recapitulates these events with cells undergoing an EMT followed by expression of mesoderm markers including Brachyury, Nodal, Wnt3, Fgf8, Mesp1 and Tbx6 [20, 127, 156].

Multiple reports have shown that disrupting Wnt activity in EpiSCs with small molecule inhibitors (XAV939, IWP-2, IWR-1) (Table 2) or genetic knockdown of $\beta$-catenin (Ctnnb1), promotes a more homogeneous primed state, enhances clonal expansion of single cells and derivation efficiency $[20,127,142$, $156,159]$, and enriches for their capacity to undergo reversion towards ESCs [127]. However, when EpiSCs are derived in the presence of Wnt inhibitors, the resulting cell lines are, for as yet unknown reasons, dependent on these enhanced culture conditions. While EpiSC lines derived in standard FA can be reversibly exposed to the Wnt pathway inhibitor IWP-2 without negative effects, EpiSCs derived with FA and IWP-2 rapidly differentiate when IWP-2 is removed [146]. The long-term effects of Wnt signaling inhibition therefore need to be investigated in more detail. Wnt-inhibited EpiSCs (WiEpiSCs) have elevated expression of E-cadherin, and decreased Snai1 and $\mathrm{N}$-cadherin (Cdh2), potentially indicating a reduction in the fraction of cells undergoing EMT. Mesoderm and endoderm markers, such as Eomes, Brachyury, FoxA2, Gata6, Sox17 and Lefty2, are downregulated and pluripotency markers are upregulated [127, 142, 146, 156, 159]. Global transcriptional analysis suggests that WiEpiSCs are similar to pre or early streak embryos [119, 127] or cells of the later Epi before entering the PS [159]. Therefore, blocking Wnt signaling inhibits differentiation, maintaining cells in a more robust state of self-renewal, akin to a developmentally advanced version of the naïve ESC "ground state" captured by FGF inhibition.

While naïve ESCs can generate chimaeras when injected into pre-implantation embryos, primed EpiSCs cannot. Although the functional potential of WiEpiSCs has been tested in chimaera assays, discrepancies in culture conditions (Table 2) and experimental design, between studies means that the data are difficult to interpret. WiEpiSCs maintained with FA and XAV939, or FGF and IWR-1, can contribute to all germ layers when grafted into post-implantation embryos [156, 159]. WiEpiSCs cultured with IWR-1 cannot contribute to pre-implantation embryos [159], but are cultured in the absence of Activin, which may limit their functional potential and the functional potential of XAV-treated cells has not been tested in pre-implantation embryos. WiEpiSCs cultured with IWP-2 have the capacity to contribute to embryonic development when injected into blastocysts [127], but their ability to contribute to postimplantation embryos has not been assessed. It therefore remains an open question as to whether these cells can contribute to both pre- and post-implantation stages of embryonic development.

Although Wnt signaling inhibition combined with FA maintains EpiSCs in a relatively stable primed pluripotent state, inhibition of Wnt signaling without FA leads to gradual differentiation $[20,156]$. Intriguingly, a combination of small molecules that simultaneously inhibit (XAV939) and activate (CHIR99021 or IWR-1) Wnt activity, prevent differentiation in the absence of FA and can be used to derive EpiSCs directly from embryos [20], although it is not known whether these cultures are homogeneous (Table 2). The exact function of this cocktail is unknown, but involves sequestering $\beta$-CATENIN in the cytoplasm independent of its activity as a transcriptional regulator. Nevertheless, $\beta$-catenin mutant cells can be maintained in FA indicating that cytoplasmic $\beta$-CATENIN is not necessary for EpiSC self-renewal under normal conditions. While CHIR/XAV EpiSCs share many transcriptional hallmarks with FA EpiSCs, the naïve markers Dppa2, Dppa4 and Dppa5a are more highly expressed and Eomes and Nodal are expressed at lower levels [20]. These cells may therefore exist in an intermediate state between EpiSCs and ESCs. CHIR/ XAV EpiSCs can differentiate in vitro into all germ layers and form teratomas in vivo. However, unlike WiEpiSCs, they cannot generate chimaeras when injected into blastocysts, and their functional potential at later developmental stages has not been assessed [20].

In addition to the promiscuous expression of lineage markers in EpiSC cultures, there is also heterogeneous expression of earlier ESC and germ layer genes, marked by an Oct4-GFP reporter [131]. The effect of Wnt signaling inhibition on this population has not been 
assessed, however, EpiSCs uniformly expressing Oct4GFP can be derived using FGF4 alone instead of FA [160] (Table 2). When the Epi of E5.5-6.5 embryos is explanted in FGF4 medium, most cells downregulate Oct4-GFP and likely differentiate but rare Oct4-GFP positive cells persist and can be purified by fluorescence activated cell sorting (FACS) [160]. Although FGF4cultured EpiSCs (F4-EpiSCs) are pluripotent, they are distinct from Oct4-GFP cells in standard FA cultures, as they show reduced naïve marker expression and high levels of lineage markers. Therefore, while an apparently homogeneous, stable EpiSC culture can be maintained in FGF4 alone, it may represent a homogeneous late anterior PS-like state, similar to that described by Kojima et al. [119] rather than an intermediate. Heterogeneous expression of Oct4-GFP is reestablished when these cells are transferred to FA but it was not determined whether this was induced by Activin, distinct activities of FGF2 versus FGF4, or the disparity in FGF concentrations used (5 ng/ml FGF2 versus $25 \mathrm{ng} / \mathrm{ml} \mathrm{FGF4).}$

\section{(iii) combining naïve and primed culture conditions}

Although Wnt signaling stimulates differentiation in the primed state of pluripotency, it stabilizes naïve pluripotency. It has therefore been suggested that culturing ESCs in a combination of primed culture conditions (FA), which normally coerce ESCs into EpiSCs, and Wnt signaling captures an intermediate state $[155,161]$. ESCs cultured in FA and CHIR exhibit a mix of naïve and primed characteristics (Table 2) [155]. Although Wnt activity has previously been shown to induce Brachyury expression correlated with EpiSC instability in FA cultures [142], surprisingly this was not the case in FA and CHIR-cultured intermediate pluripotent stem cells (INTPSCs) [155]. INTPSCs retain ESC features including domed colony morphology, high clonogenicity, naïve marker expression (e.g. Klf4, Rex1 and Esrrb), X chromosome activation and the capacity to contribute to embryonic development when injected into blastocysts [155]. They also acquire some EpiSC-like features such as the emergence of a subpopulation of cells coexpressing pluripotency (OCT4, ESRRB) and germ layer (FOXA2) markers. However, the majority of primed Epi markers, including Fgf5, Wnt3 and Otx2, are expressed at levels intermediate between ESCs and EpiSCs. Although attempts were not made to derive INTPSCs directly from embryos, PSC lines have been derived from pre-implantation embryos under similar conditions with FA and a comparable Wnt pathway agonist, BIO with the addition of a LIF blocking antibody [161] (Table 2). While FGF, Activin, BIO stem cells (FAB-SCs) express intermediate levels Fgf5, associated with the primed state, and naïve miRNAs, they do not express other naïve markers at appreciable levels and are morphologically more similar to EpiSCs [161]. Furthermore, FAB-SCs are not functionally pluripotent as assessed by in vitro differentiation, teratoma assays and blastocyst injections [161]. While their capacity to generate teratomas could be rescued by LIF and BMP, it was not determined whether these cells had reverted to an ESC-like state.

Similar attempts were made to culture ESCs in the presence of the conventional ESC propagation component, serum (containing BMP activity), alongside the primed culture component Activin. Clonal assays selected for rare ESCs that can be maintained in an undifferentiated state in serum and Activin with similar efficiency to SL [162]. As forced expression of Nanog is sufficient to maintain self-renewal in the absence of LIF $[74,75]$ and Nanog is a direct target of Activin/Nodal signaling, this may explain how Activin maintains pluripotency in this context. These cells occupy a transcriptional midpoint between ESCs and EpiSCs expressing intermediate levels of naïve markers (Rex1, Stella, Klf4, Nanog) and primed/lineage markers (Fgf5, Nodal, Lefty, FoxA2, Otx2, Gata6, Brachyury), and are hence referred to as intermediate ESCs (IESCs) (Table 2). While not related to standard ESC or EpiSC culture conditions, a transcriptionally similar state can be generated by longterm culture of ESCs in conditioned medium (MEDII) from a human hepatocellular carcinoma cell line (HepG2) (Table 2). In contrast to IESCs which display a compact domed colony morphology [162], these early primitive ectoderm-like (EPL) cells have a flattened EpiSC-like morphology [163]. While EPL cells maintain the expression of some naïve markers including SSEA-1 and Alkaline Phosphatase, they downregulate others including Rex1 and Gbx2. Furthermore, they upregulate the early post-implantation Epi marker, Fgf5, but not later PS or lineage-associated genes. Transferring IESCs or EPL cells to SL medium regenerates a standard ESC transcriptional profile indicating that these cells are not committed to their states. Neither IESCs nor EPL cells can contribute to embryonic development when introduced into pre-implantation embryos but their capacity to contribute to post-implantation development has not been assessed.

\section{Human pluripotent stem cells}

As for mouse, multiple human states of pluripotency have been captured in vitro. Initial attempts to derive human ESCs (hESCs) by explanting the blastocyst ICM in mouse ESC (mESC) self-renewal conditions, SL, were unsuccessful [164, 165]. Instead, like EpiSCs, hESCs rely on FGF and Activin for self-renewal, exhibit a flattened colony morphology, limited clonogenicity, preferential use of the Oct4 proximal enhancer and reduced expression of the naïve markers REX1 and TFCP2L1 compared 
to the human blastocyst ICM $[3,17,125,127,166-171]$. The $\mathrm{X}$ chromosome activation status varies between hESC lines, perhaps a result of inconsistency in the oxygen levels in different derivation protocols [166, 171, 172]. Furthermore, hESC cultures experience high levels of spontaneous differentiation and contain subpopulations that coexpress pluripotency and lineage markers including BRACHYURY and GATA4 [127, 173]. As with EpiSCs, Wnt pathway inhibition increases hESC homogeneity and reduces differentiation $[127,159]$. Taken together, these findings indicate that the first human PSC state to be derived corresponds to a primed state of pluripotency. This begs the question as to whether nomenclature should now be re-aligned, and human ESCs be classified as human EpiSCs.

Initially it was not known whether a naïve human pluripotent state existed. One possibility being that this state is uniquely stabilized and more accessible in rodents due to their capacity to undergo diapause. Culturing hESCs in mESC ground state conditions (2i and LIF) results in extensive differentiation [174-177]. However, multiple modified small molecule inhibitor cocktails have now been identified that can induce naïve properties in hESCs [175-182], some of which also facilitate the derivation of cell lines directly from human embryos [175-177, 181]. Purported naïve hESCs share common phenotypic properties that differ from canonical primed hESCs including domed colony morphology, resistance to single cell dissociation, enhanced proliferation rate, increased $\mathrm{X}$ activation and decreased methylation at naïve loci [175-182]. Not surprisingly, the various, distinct combinations of inhibitors yield hESC states with vastly different transcriptional profiles. While several of these states transcriptionally cluster with naïve mESCs, rather than primed EpiSCs or hESCs [175-177], others are distinct from pluripotent states existing in the mouse. For example, Chan et al. [178] describe hESCs that upregulate the expression of genes associated with naïve pluripotency in mouse (KLF2, KLF4, KLF5, $D P P A 3, D P P A 5$ and NANOG) but also significantly upregulate a long list of lineage-associated markers (HNF4 $\alpha$, GATA6, GATA4, SOX17, FOXA2, T, EOMES, GSC, CDX2, WNT3, CDX2 and NODAL) and contain a fraction of cells that coexpress GATA6 and NANOG protein. Additionally, the majority of these cultures require FGF and Activin/Nodal signaling, and hence may be more similar to novel intermediate states of pluripotency described in mouse than bona fide naïve pluripotent cells. However, FGF and Activin signaling independence has been attained through genetic manipulation of hESC lines by forced continuous activation of STAT3 [183] or transient expression of NANOG and KLF2 [184]. Notably, culture conditions comprising of the $2 \mathrm{i}$ inhibitors [91] alongside an inhibitor of protein kinase C (PKC) [184] can be used to successfully derive novel hESC lines in FGF and Activin independent conditions directly from embryos [181, 184].

Both naïve and primed hESCs generate derivatives of all embryonic germ layers when differentiated in vitro, or in vivo in teratoma assays [185-187]. Perhaps not surprisingly, naïve hESC states exhibit a reduced capacity to form mature cell types compared to primed hESCs when challenged with the same differentiation protocols. [188]. This is likely due to the fact that, for efficient differentiation, they must recapitulate the in vivo developmental trajectory and first transit to a postimplantation primed Epi state. While there are suggestions that hESCs generate trophoblast derivatives [127, 189-192], many of the genes used to define this cell type are also expressed in other lineages [193], leaving this issue unresolved. To determine whether hESCs maintain the capacity to contribute to embryonic development after periods in culture, they have also been introduced into mouse embryos. When primed hESCs are injected into mouse blastocysts, they persist at postimplantation stages, but negatively affect embryonic development [194]. Generally, hESCs also show poor contribution when grafted into post-implantation mouse embryos, but this can be improved by culturing hESCs in the presence of Wnt signaling inhibitors [159]. The capacity of naïve hESCs to contribute to preimplantation mouse embryos is still unclear. While in some cases contribution has been observed, there is evidence that this is not reproducible and cells do not integrate $[175,176,184,195]$. However, this is by no means an infallible experiment and potential interspecies incompatibility means that these results are difficult to interpret.

While hESCs share many properties with mouse EpiSCs, akin to the post-implantation Epi around gastrulation, they also show a strong correlation with EpiLCs, similar to the Epi at peri- or early postimplantation stages of development [196]. However, without access to post-implantation human embryos, there are limitations on temporally aligning human PSCs to in vivo development. Attempts have been made to correlate human states of pluripotency with mouse development, but transcriptional differences between species [197-199] suggest that this may not be the optimal approach. Nevertheless, single-cell RNA-sequencing of embryos from the more closely related cynomolgous monkey revealed that standard hESCs are indeed similar to the primate postimplantation Epi, while naïve hESCs show a stronger correlation with the primate pre-implantation Epi [196]. Recent advances in the ex vivo culture of human embryos allow development to implantation-like stages [200, 201], and therefore may also provide new 
insights into later human development. However, since there is no possibility for in vivo validation, conclusions should be drawn tentatively.

\section{Review and conclusions}

While pluripotent cells are present in the embryo for a significant period of time during the course of development, for the past decade, in vitro PSCs have been limited to naïve ESCs and primed EpiSCs, perhaps suggesting that only a restricted number of stable attractor states can be isolated and stably maintained. However, recent developments in the field have revealed that these states can interconvert in vitro via distinct transient intermediates, hence the view of pluripotency as a continuum. Furthermore, modified culture conditions can induce novel characteristics in ESCs or EpiSCs confirming that the functional definition of pluripotency can be fulfilled while encompassing a broad spectrum of additional properties. What remains unclear is how these newly described states relate to one another, and whether bona fide counterparts exist in the embryo, neither of which are trivial questions to answer. Our current understanding is based on data generated using a range of basal media, cytokine combinations, cytokine concentrations, functional assays and transcriptional analysis platforms making direct comparisons near impossible. To understand whether the differences between these states are representative of an endogenous developmental progression, one needs to determine how each state functionally and transcriptionally relates to another in parallel controlled experiments, followed by comparisons to high-resolution data from the Epi population through successive stages of embryonic development. Ideally, these comparisons would be at a single-cell level, as heterogeneity within cell cultures, as well as in vivo within the Epi itself, may otherwise make these data challenging to interpret. The increase in the availability of single-cell data from both pre and postimplantation embryos suggests that this may soon be possible [202-206]. Furthermore, one must keep in mind that in vivo pluripotency is not a stable state, and there is no such thing as self-renewal, hence in vitro imitations will inevitably exhibit a certain degree of disparity.

\footnotetext{
Abbreviations

2i: 2 inhibitor medium; DE: Distal enhancer; E: Embryonic day; EB: Embryoid body; EMT: Epithelial to mesenchymal transition; Epi: Epiblast; EpiLCs: Epiblast-like cells; EpiSCs: Epiblast stem cells; EPL: Early primitive ectoderm-like cells; ESCs: Embryonic stem cells; ESD-EpiSC: ESC-derived epiblast stem cells; F4-EpiSCs: FGF4-EpiSCs; FA: FGF2 and Activin A; FABSCs: FGF, Activin, BIO stem cells; GLT: Germline transmission; hESCs: Human embryonic stem cells; ICM: Inner cell mass; iESCs: Intermediate embryonic stem cells; INTPSCs: Intermediate pluripotent stem cells; KOSR: Knockout serum replacement; mESCs: Mouse embryonic stem cells; miRNAs: micro RNAs; PE: Proximal enhancer; PGCLCs: PGC-like cells; PGCs: Primordial germ cells; PrE: Primitive endoderm; PS: Primitive streak; PSC: Pluripotent stem cell; rESCs: Reprogrammed Epi ESC-like cells; SL: Serum and LIF; VE: Visceral endoderm; WiEpiSCs: Wnt inhibited EpiSCs
}

\section{Acknowledgements}

The authors would like to thank the entire AKH lab for critical discussion of the manuscript. SMM is supported by a Wellcome Trust Sir Henry Wellcome Postdoctoral Fellowship. Work in AKH's lab is supported by the National Institutes of Health (R01DK084391, P30CA008748), and the Tri-Institutional Stem Cell Initiative supported by The Starr Foundation. Work in JN's lab is supported by the Wellcome Trust, Medical Research Council (MRC), Biotechnology and Biological Sciences Research Council and a core support grant from the Wellcome Trust and MRC to the Wellcome Trust - Medical Research Council Cambridge Stem Cell Institute.

\section{Author's contribution}

SMM and AKH conceived of and wrote the manuscript. JN provided detailed feedback. All authors read and approved the final manuscript.

\section{Competing interests}

The authors declare they have no competing interests.

\section{Author details}

${ }^{1}$ Developmental Biology Program, Sloan Kettering Institute, Memorial Sloan Kettering Cancer Center, New York, NY 10065, USA. ²Wellcome Trust-Medical Research Council Centre for Stem Cell Research, University of Cambridge, Tennis Court Road, Cambridge CB2 1QR, UK.

Received: 28 March 2017 Accepted: 1 June 2017

Published online: 13 June 2017

\section{References}

1. Arnold SJ, Robertson EJ. Making a commitment: cell lineage allocation and axis patterning in the early mouse embryo. Nat Rev Mol Cell Biol. 2009;10(2):91-103.

2. Nichols J, Smith A. Naive and primed pluripotent states. Cell Stem Cell. 2009:4(6):487-92.

3. Brons IG, et al. Derivation of pluripotent epiblast stem cells from mammalian embryos. Nature. 2007:448(7150):191-5.

4. Evans MJ, Kaufman MH. Establishment in culture of pluripotential cells from mouse embryos. Nature. 1981;292(5819):154-6.

5. Kaufman-Francis, K., et al., Differential response of epiblast stem cells to Nodal and Activin signalling: a paradigm of early endoderm development in the embryo. Philos Trans R Soc Lond B Biol Sci, 2014. 369(1657).

6. Tesar PJ. Derivation of germ-line-competent embryonic stem cell lines from preblastocyst mouse embryos. Proc Natl Acad Sci U S A. 2005;102(23):8239-44.

7. Doetschman TC, et al. The in vitro development of blastocyst-derived embryonic stem cell lines: formation of visceral yolk sac, blood islands and myocardium. J Embryol Exp Morphol. 1985;87:27-45.

8. Keller GM. In vitro differentiation of embryonic stem cells. Curr Opin Cell Biol. 1995; 7(6):862-9.

9. Leahy $\mathrm{A}$, et al. Use of developmental marker genes to define temporal and spatial patterns of differentiation during embryoid body formation. J Exp Zool. 1999;284(1):67-81.

10. Beddington RS, Robertson EJ. An assessment of the developmental potential of embryonic stem cells in the midgestation mouse embryo. Development. 1989;105(4):733-7.

11. Huang $Y$, et al. In vivo differentiation potential of epiblast stem cells revealed by chimeric embryo formation. Cell Rep. 2012;2(6):1571-8.

12. Martin GR. Isolation of a pluripotent cell line from early mouse embryos cultured in medium conditioned by teratocarcinoma stem cells. Proc Nat Acad Sci U S A. 1981;78(12):7634-8.

13. Boroviak $T$, et al. The ability of inner-cell-mass cells to self-renew as embryonic stem cells is acquired following epiblast specification. Nat Cell Biol. 2014;16(6):516-28.

14. Brook FA, Gardner RL. The origin and efficient derivation of embryonic stem cells in the mouse. Proc Natl Acad Sci U S A. 1997:94(11):5709-12.

15. Delhaise F, et al. Establishment of an embryonic stem cell line from 8-cell stage mouse embryos. Eur J Morphol. 1996;34(4):237-43.

16. Guo G, et al. Klf4 reverts developmentally programmed restriction of ground state pluripotency. Development. 2009;136(7):1063-9.

17. Tesar PJ, et al. New cell lines from mouse epiblast share defining features with human embryonic stem cells. Nature. 2007;448(7150):196-9.

18. Bao $S$, et al. Epigenetic reversion of post-implantation epiblast to pluripotent embryonic stem cells. Nature. 2009;461(7268):1292-5. 
19. Hanna J, et al. Metastable pluripotent states in NOD-mouse-derived ESCs. Cell Stem Cell. 2009;4(6):513-24.

20. Kim H, et al. Modulation of beta-catenin function maintains mouse epiblast stem cell and human embryonic stem cell self-renewal. Nat Commun. 2013; 4:2403.

21. Bernemann C, et al. Distinct developmental ground states of epiblast stem cell lines determine different pluripotency features. Stem Cells. 2011;29(10): 1496-503.

22. Greber B, et al. Conserved and divergent roles of FGF signaling in mouse epiblast stem cells and human embryonic stem cells. Cell Stem Cell. 2010; 6(3):215-26.

23. Zhou H, et al. Conversion of mouse epiblast stem cells to an earlier pluripotency state by small molecules. J Biol Chem. 2010;285(39):29676-80

24. Yang J, et al. Stat3 activation is limiting for reprogramming to ground state pluripotency. Cell Stem Cell. 2010;7(3):319-28.

25. Silva J, et al. Nanog is the gateway to the pluripotent ground state. Cell. 2009:138(4):722-37.

26. Guo G, Smith A. A genome-wide screen in EpiSCs identifies Nr5a nuclear receptors as potent inducers of ground state pluripotency. Development. 2010;137(19):3185-92.

27. Smith $A G$, et al. Inhibition of pluripotential embryonic stem cell differentiation by purified polypeptides. Nature. 1988;336(6200):688-90.

28. Williams RL, et al. Myeloid leukaemia inhibitory factor maintains the developmental potential of embryonic stem cells. Nature. 1988;336(6200):684-7.

29. Ying $\mathrm{QL}$, et al. BMP induction of id proteins suppresses differentiation and sustains embryonic stem cell self-renewal in collaboration with STAT3. Cell. 2003;115(3):281-92.

30. Ohtsuka S, Nakai-Futatsugi Y, Niwa H. LIF signal in mouse embryonic stem cells. JAKSTAT. 2015;4(2):e1086520.

31. Morikawa M, et al. BMP sustains embryonic stem cell self-renewal through distinct functions of different Kruppel-like factors. Stem Cell Reports. 2016; 6(1):64-73.

32. Conover JC, et al. Ciliary neurotrophic factor maintains the pluripotentiality of embryonic stem cells. Development. 1993;119(3):559-65.

33. Pennica D, et al. Cardiotrophin-1. Biological activities and binding to the leukemia inhibitory factor receptor/gp130 signaling complex. J Biol Chem. 1995;270(18):10915-22.

34. Rose TM, et al. Oncostatin M (OSM) inhibits the differentiation of pluripotent embryonic stem cells in vitro. Cytokine. 1994;6(1):48-54.

35. Wolf $\mathrm{E}_{\text {, et }}$ al. Efficient generation of chimaeric mice using embryonic stem cells after long-term culture in the presence of ciliary neurotrophic factor. Transgenic Res. 1994;3(3):152-8.

36. Yoshida K, et al. Maintenance of the pluripotential phenotype of embryonic stem cells through direct activation of gp130 signalling pathways. Mech Dev. 1994;45(2):163-71.

37. Hirai $H$, Karian P, Kikyo N. Regulation of embryonic stem cell self-renewal and pluripotency by leukaemia inhibitory factor. Biochem J. 2011;438(1):11-23.

38. Li Z, et al. BMP4 signaling acts via dual-specificity phosphatase 9 to control ERK activity in mouse embryonic stem cells. Cell Stem Cell. 2012:10(2):171-82.

39. Qi X, et al. BMP4 supports self-renewal of embryonic stem cells by inhibiting mitogen-activated protein kinase pathways. Proc Natl Acad Sci U S A. 2004;101(16):6027-32.

40. Di-Gregorio A, et al. BMP signalling inhibits premature neural differentiation in the mouse embryo. Development. 2007;134(18):3359-69.

41. Ware $\mathrm{CB}$, et al. Targeted disruption of the low-affinity leukemia inhibitory factor receptor gene causes placental, skeletal, neural and metabolic defects and results in perinatal death. Development. 1995;121(5):1283-99.

42. Takeda K, et al. Targeted disruption of the mouse Stat3 gene leads to early embryonic lethality. Proc Natl Acad Sci U S A. 1997;94(8):3801-4.

43. Nichols J, et al. Physiological rationale for responsiveness of mouse embryonic stem cells to gp130 cytokines. Development. 2001;128(12):2333-9.

44. Li M, Sendtner M, Smith A. Essential function of LIF receptor in motor neurons. Nature. 1995;378(6558):724-7.

45. Do DV, et al. A genetic and developmental pathway from STAT3 to the OCT4-NANOG circuit is essential for maintenance of ICM lineages in vivo. Genes Dev. 2013;27(12):1378-90.

46. Martin Gonzalez J, et al. Embryonic stem cell culture conditions support distinct states associated with different developmental stages and potency. Stem Cell Reports. 2016;7(2):177-91.

47. Ahmed, K., et al., Global Chromatin Architecture Reflects Pluripotency and Lineage Commitment in the Early Mouse Embryo. PLoS One, 2010. 5(5).
48. Efroni S, et al. Global transcription in pluripotent embryonic stem cells. Cell Stem Cell. 2008;2(5):437-47.

49. Gaspar-Maia, A., et al., Open chromatin in pluripotency and reprogramming (vol 12, pg 36, 2011). Nat Rev Mol Cell Biol, 2011. 12(4).

50. Aoto T, et al. Nuclear and chromatin reorganization in the MHC-Oct3/4 locus at developmental phases of embryonic stem cell differentiation. Dev Biol. 2006;298(2):354-67.

51. Azuara V, et al. Chromatin signatures of pluripotent cell lines. Nat Cell Biol. 2006;8(5):532-8

52. Meissner A, et al. Genome-scale DNA methylation maps of pluripotent and differentiated cells. Nature. 2008;454(7205):766-70.

53. Christophorou MA, et al. Citrullination regulates pluripotency and histone $\mathrm{H1}$ binding to chromatin. Nature. 2014;507(7490):104-+.

54. Xiao $\mathrm{S}$, et al. SMARCAD1 contributes to the regulation of naive Pluripotency by interacting with Histone Citrullination. Cell Rep. 2017:18(13):3117-28.

55. Williams LH, et al. Transcription precedes loss of Xist coating and depletion of H3K27me3 during X-chromosome reprogramming in the mouse inner cell mass. Development. 2011;138(10):2049-57.

56. Chen $\mathrm{G}$, et al. Single-cell analyses of $\mathrm{X}$ chromosome inactivation dynamics and pluripotency during differentiation. Genome Res. 2016;26(10):1342-54

57. Yeo JC, $\mathrm{Ng} \mathrm{HH}$. The transcriptional regulation of pluripotency. Cell Res. 2013;23(1):20-32.

58. Nichols J, Smith A. Pluripotency in the embryo and in culture. Cold Spring Harb Perspect Biol. 2012;4(8):a008128.

59. Yeom Yl, et al. Germline regulatory element of Oct-4 specific for the totipotent cycle of embryonal cells. Development. 1996;122(3):881-94.

60. Houbaviy HB, Murray MF, Sharp PA. Embryonic stem cell-specific MicroRNAs. Dev Cell. 2003;5(2):351-8.

61. Marson A, et al. Connecting microRNA genes to the core transcriptional regulatory circuitry of embryonic stem cells. Cell. 2008;134(3):521-33.

62. Svoboda P, Flemr M. The role of miRNAs and endogenous siRNAs in maternal-to-zygotic reprogramming and the establishment of pluripotency. EMBO Rep. 2010;11(8):590-7.

63. Takahashi K, Yamanaka S. A decade of transcription factor-mediated reprogramming to pluripotency. Nat Rev Mol Cell Biol. 2016;17(3): 183-93.

64. Anokye-Danso F, et al. Highly efficient miRNA-mediated reprogramming of mouse and human somatic cells to Pluripotency (vol 8, pg 376, 2011). Cell Stem Cell. 2012;11(6):53-853.

65. Judson RL, et al. Embryonic stem cell-specific microRNAs promote induced pluripotency. Nat Biotechnol. 2009;27(5):459-61.

66. Melton $\mathrm{C}$, Judson RL, Blelloch R. Opposing microRNA families regulate self-renewal in mouse embryonic stem cells (vol 463, pg 621, 2010). Nature. 2010:464(7285):126.

67. Davenport TG, Jerome-Majewska LA, Papaioannou VE. Mammary gland, limb and yolk sac defects in mice lacking Tbx3, the gene mutated in human ulnar mammary syndrome. Development. 2003;130(10):2263-73.

68. Luo J, et al. Placental abnormalities in mouse embryos lacking the orphan nuclear receptor ERR-beta. Nature. 1997;388(6644):778-82.

69. Alder $\mathrm{O}$, et al. Ring1B and Suv39h1 delineate distinct chromatin states at bivalent genes during early mouse lineage commitment. Development 2010;137(15):2483-92.

70. Dahl JA, et al. Histone $\mathrm{H} 3$ lysine 27 methylation asymmetry on developmentally-regulated promoters distinguish the first two lineages in mouse preimplantation embryos. PLoS One. 2010;5(2):e9150.

71. Rugg-Gunn PJ, et al. Distinct histone modifications in stem cell lines and tissue lineages from the early mouse embryo. Proc Natl Acad Sci U S A. 2010;107(24):10783-90.

72. Tang F, et al. Tracing the derivation of embryonic stem cells from the inner cell mass by single-cell RNA-Seq analysis. Cell Stem Cell. 2010;6(5):468-78.

73. Kumar RM, et al. Deconstructing transcriptional heterogeneity in pluripotent stem cells. Nature. 2014;516(7529):56-61.

74. Chambers I, et al. Functional expression cloning of Nanog, a pluripotency sustaining factor in embryonic stem cells. Cell. 2003;113(5):643-55.

75. Mitsui $K$, et al. The homeoprotein Nanog is required for maintenance of pluripotency in mouse epiblast and ES cells. Cell. 2003;113(5):631-42.

76. Toyooka $Y$, et al. Identification and characterization of subpopulations in undifferentiated ES cell culture. Development. 2008;135(5):909-18.

77. Canham MA, et al. Functional heterogeneity of embryonic stem cells revealed through translational amplification of an early endodermal transcript. PLoS Biol. 2010;8(5):e1000379. 
78. Hayashi K, et al. Dynamic equilibrium and heterogeneity of mouse pluripotent stem cells with distinct functional and epigenetic states. Cell Stem Cell. 2008;3(4):391-401.

79. Falco G, et al. Zscan4: a novel gene expressed exclusively in late 2-cell embryos and embryonic stem cells. Dev Biol. 2007;307(2):539-50.

80. Macfarlan TS, et al. Embryonic stem cell potency fluctuates with endogenous retrovirus activity. Nature. 2012;487(7405):57-63.

81. De laco, A., et al., DUX-family transcription factors regulate zygotic genome activation in placental mammals. Nat Genet, 2017.

82. Zalzman $\mathrm{M}$, et al. Zscan4 regulates telomere elongation and genomic stability in ES cells. Nature. 2010;464(7290):858-63.

83. Amano, T., et al., Zscan4 restores the developmental potency of embryonic stem cells. Nat Commun. 2013;4:1966. doi:10.1038/ncomms2966.

84. Nakai-Futatsugi Y, Niwa H. Zscan4 is activated after telomere shortening in mouse embryonic stem cells. Stem Cell Reports. 2016;6(4):483-95.

85. Plusa $B$, et al. Distinct sequential cell behaviours direct primitive endoderm formation in the mouse blastocyst. Development. 2008; 135(18):3081-91.

86. Chazaud $C$, et al. Early lineage segregation between epiblast and primitive endoderm in mouse blastocysts through the Grb2-MAPK pathway. Dev Cell. 2006;10(5):615-24.

87. Xenopoulos $\mathrm{P}$, et al. Heterogeneities in Nanog expression drive stable commitment to Pluripotency in the mouse Blastocyst. Cell Rep. 2015;10(9):1508-1520.

88. Guo GJ, et al. Serum-based culture conditions provoke Gene expression variability in mouse embryonic stem cells as revealed by single-cell analysis. Cell Rep. 2016;14(4):956-65.

89. Kunath $T$, et al. FGF stimulation of the Erk1/2 signalling cascade triggers transition of pluripotent embryonic stem cells from self-renewal to lineage commitment. Development. 2007;134(16):2895-902.

90. Silva J, Smith A. Capturing pluripotency. Cell. 2008;132(4):532-6.

91. Ying $\mathrm{QL}$, et al. The ground state of embryonic stem cell self-renewal. Nature. 2008:453(7194):519-23.

92. Wray J, et al. Inhibition of glycogen synthase kinase-3 alleviates Tcf3 repression of the pluripotency network and increases embryonic stem cell resistance to differentiation. Nat Cell Biol. 2011;13(7):838-45.

93. Merrill BJ. Wnt pathway regulation of embryonic stem cell self-renewal. Cold Spring Harb Perspect Biol. 2012;4(9):a007971.

94. ten Berge $D$, et al. Embryonic stem cells require Wnt proteins to prevent differentiation to epiblast stem cells. Nat Cell Biol. 2011;13(9):1070-5.

95. Marks $\mathrm{H}$, et al. The transcriptional and epigenomic foundations of ground state pluripotency. Cell. 2012;149(3):590-604.

96. Kolodziejczyk AA, et al. Single cell RNA-sequencing of Pluripotent states unlocks modular transcriptional variation. Cell Stem Cell. 2015;17(4):471-85

97. Morgani SM, et al. Totipotent embryonic stem cells arise in ground-state culture conditions. Cell Rep. 2013;3(6):1945-57.

98. Schugar RC, Robbins PD, Deasy BM. Small molecules in stem cell selfrenewal and differentiation. Gene Ther. 2008;15(2):126-35.

99. Efe JA, Ding S. The evolving biology of small molecules: controlling cell fate and identity. Philos Trans R Soc Lond Ser B Biol Sci. 2011;366(1575):2208-21.

100. Dutta $D$, et al. Self-renewal versus lineage commitment of embryonic stem cells: protein kinase C signaling shifts the balance. Stem Cells. 2011; 29(4):618-28.

101. Ward CM, et al. Efficient germline transmission of mouse embryonic stem cells grown in synthetic serum in the absence of a fibroblast feeder layer. Lab Investig. 2002;82(12):1765-7.

102. Blaschke $K$, et al. Vitamin C induces Tet-dependent DNA demethylation and a blastocyst-like state in ES cells. Nature. 2013;500(7461):222-6.

103. Papatsenko D, et al. Single-cell analyses of ESCS reveal alternative Pluripotent cell states and molecular mechanisms that control self-renewal. Stem Cell Reports. 2015;5(2):207-20.

104. Ying QL, et al. Conversion of embryonic stem cells into neuroectodermal precursors in adherent monoculture. Nat Biotechnol. 2003;21(2):183-6.

105. Hamazaki T, et al. Aggregation of embryonic stem cells induces Nanog repression and primitive endoderm differentiation. J Cell Sci. 2004;117(Pt 23):5681-6.

106. Price FD, et al. Canonical Wnt signaling induces a primitive endoderm metastable state in mouse embryonic stem cells. Stem Cells. 2013;31(4): 752-64.

107. Lancaster MA, Knoblich JA. Organogenesis in a dish: modeling development and disease using organoid technologies. Science. 2014; 345(6194):1247125.
108. Turner DA, Baillie-Johnson P, Martinez Arias A. Organoids and the genetically encoded self-assembly of embryonic stem cells. BioEssays. 2016: 38(2):181-91.

109. Huang J, et al. Efficient production of mice from embryonic stem cells injected into four- or eight-cell embryos by piezo micromanipulation. Stem Cells. 2008:26(7):1883-90

110. Poueymirou WT, et al. Fo generation mice fully derived from gene-targeted embryonic stem cells allowing immediate phenotypic analyses. Nat Biotechnol. 2007;25(1):91-9.

111. Saburi $\mathrm{S}$, et al. Developmental fate of single embryonic stem cells microinjected into 8-cell-stage mouse embryos. Differentiation. 1997; 62(1):1-11.

112. Lallemand $Y$, Brulet $P$. An in situ assessment of the routes and extents of colonisation of the mouse embryo by embryonic stem cells and their descendants. Development. 1990;110(4):1241-8.

113. Eakin GS, et al. Developmental potential and behavior of tetraploid cells in the mouse embryo. Dev Biol. 2005;288(1):150-9.

114. Nagy A, et al. Embryonic stem cells alone are able to support fetal development in the mouse. Development. 1990;110(3):815-21.

115. Nagy A, et al. Derivation of completely cell culture-derived mice from earlypassage embryonic stem cells. Proc Natl Acad Sci U S A. 1993;90(18):8424-8

116. Eggan K, et al. Hybrid vigor, fetal overgrowth, and viability of mice derived by nuclear cloning and tetraploid embryo complementation. Proc Natl Acad Sci U S A. 2001;98(11):6209-14.

117. Hayashi K, Saitou M. Perspectives of germ cell development in vitro in mammals. Anim Sci J. 2014;85(6):617-26.

118. Osorno R, et al. The developmental dismantling of pluripotency is reversed by ectopic Oct4 expression. Development. 2012;139(13):2288-98.

119. Kojima $Y$, et al. The transcriptional and functional properties of mouse epiblast stem cells resemble the anterior primitive streak. Cell Stem Cell. 2014;14(1):107-20

120. Najm FJ, et al. Isolation of epiblast stem cells from preimplantation mouse embryos. Cell Stem Cell. 2011:8(3):318-25.

121. Tosolini $M$, Jouneau A. From naive to primed Pluripotency: in vitro conversion of mouse embryonic stem cells in Epiblast stem cells. Methods Mol Biol. 2016;1341:209-16.

122. Turco MY, et al. Cellular heterogeneity during embryonic stem cell differentiation to epiblast stem cells is revealed by the ShcD/RaLP adaptor protein. Stem Cells. 2012;30(11):2423-36.

123. Camus A, et al. Absence of Nodal signaling promotes precocious neural differentiation in the mouse embryo. Dev Biol. 2006;295(2):743-55.

124. Mesnard D, Guzman-Ayala M, Constam DB. Nodal specifies embryonic visceral endoderm and sustains pluripotent cells in the epiblast before overt axial patterning. Development. 2006;133(13):2497-505.

125. Vallier $L$, et al. Activin/Nodal signalling maintains pluripotency by controlling Nanog expression. Development. 2009;136(8):1339-49.

126. Feldman B, et al. Requirement of FGF-4 for postimplantation mouse development. Science. 1995;267(5195):246-9.

127. Kurek D, et al. Endogenous WNT signals mediate BMP-induced and spontaneous differentiation of epiblast stem cells and human embryonic stem cells. Stem Cell Reports. 2015;4(1):114-28.

128. Watanabe $K$, et al. A ROCK inhibitor permits survival of dissociated human embryonic stem cells. Nat Biotechnol. 2007;25(6):681-6.

129. Hunt GC, Singh P, Schwarzbauer JE. Endogenous production of fibronectin is required for self-renewal of cultured mouse embryonic stem cells. Exp Cell Res. 2012;318(15):1820-31.

130. Veluscek $\mathrm{G}$, et al. Jun-mediated changes in cell adhesion contribute to mouse embryonic stem cell exit from ground state Pluripotency. Stem Cells. 2016;34(5):1213-24.

131. Han DW, et al. Epiblast stem cell subpopulations represent mouse embryos of distinct pregastrulation stages. Cell. 2010;143(4):617-27.

132. Gillich $A$, et al. Epiblast stem cell-based system reveals reprogramming synergy of germline factors. Cell Stem Cell. 2012;10(4):425-39.

133. Jouneau $A$, et al. Naive and primed murine pluripotent stem cells have distinct miRNA expression profiles. RNA. 2012;18(2):253-64.

134. Hart AH, et al. Identification, cloning and expression analysis of the pluripotency promoting Nanog genes in mouse and human. Dev Dyn. 2004;230(1):187-98.

135. Saiz N, et al. Asynchronous fate decisions by single cells collectively ensure consistent lineage composition in the mouse blastocyst. Nat Commun. 2016;7:13463. 
136. Song J, et al. DNA and chromatin modification networks distinguish stem cell Pluripotent ground states. Mol Cell Proteomics. 2012;11(10):1036-47.

137. Orkin SH, Hochedlinger K. Chromatin connections to pluripotency and cellular reprogramming. Cell. 2011;145(6):835-50.

138. Factor DC, et al. Epigenomic comparison reveals activation of "seed" enhancers during transition from naive to primed pluripotency. Cell Stem Cell. 2014;14(6):854-63.

139. Zhang $H$, et al. MLL1 inhibition reprograms Epiblast stem cells to naive Pluripotency. Cell Stem Cell. 2016;18(4):481-94.

140. Veillard AC, et al. Stable Methylation at promoters distinguishes Epiblast stem cells from embryonic stem cells and the in vivo Epiblasts. Stem Cells Dev. 2014;23(17):2014-29.

141. Iwafuchi-Doi M, et al. Transcriptional regulatory networks in epiblast cells and during anterior neural plate development as modeled in epiblast stem cells. Development. 2012;139(21):3926-37.

142. Tsakiridis A, et al. Distinct Wnt-driven primitive streak-like populations reflect in vivo lineage precursors. Development. 2014;141(6):1209-21.

143. Sun B, et al. Status of genomic imprinting in epigenetically distinct pluripotent stem cells. Stem Cells. 2012;30(2):161-8.

144. Mak W, et al. Reactivation of the paternal X chromosome in early mouse embryos. Science. 2004;303(5658):666-9.

145. Hayashi $K$, et al. Reconstitution of the mouse germ cell specification pathway in culture by pluripotent stem cells. Cell. 2011;146(4):519-32

146. Sugimoto $M$, et al. A simple and robust method for establishing homogeneous mouse epiblast stem cell lines by wnt inhibition. Stem Cell Reports. 2015;4(4):744-57.

147. Hayashi K, Surani MA. Self-renewing epiblast stem cells exhibit continual delineation of germ cells with epigenetic reprogramming in vitro. Development. 2009;136(21):3549-56.

148. Gardner RL, et al. Clonal analysis of X-chromosome inactivation and the origin of the germ line in the mouse embryo. J Embryol Exp Morphol. 1985;88:349-63.

149. Ohtsuka S, Nishikawa-Torikai S, Niwa H. E-cadherin promotes incorporation of mouse epiblast stem cells into normal development. PLoS One. 2012;7(9):e45220.

150. Ohinata $Y$, et al. A signaling principle for the specification of the germ cell lineage in mice. Cell. 2009;137(3):571-84.

151. Kalkan, T. and A. Smith, Mapping the route from naive pluripotency to lineage specification. Philos Trans R Soc Lond B Biol Sci. 2014 Dec 5;369(1657). pii: 20130540. doi:10.1098/rstb.2013.0540.

152. Smith A. Formative pluripotency: the executive phase in a developmental continuum. Development. 2017;144(3):365-73.

153. Sancho $M$, et al. Competitive interactions eliminate unfit embryonic stem cells at the onset of differentiation. Dev Cell. 2013;26(1):19-30.

154. Gouti M, et al. In vitro generation of neuromesodermal progenitors reveals distinct roles for wnt signalling in the specification of spinal cord and paraxial mesoderm identity. PLoS Biol. 2014;12(8):e1001937.

155. Tsukiyama T, Ohinata Y. A modified EpiSC culture condition containing a GSK3 inhibitor can support germline-competent pluripotency in mice. PLoS One. 2014;9(4):e95329.

156. Sumi T, et al. Epiblast ground state is controlled by canonical Wnt/betacatenin signaling in the postimplantation mouse embryo and epiblast stem cells. PLoS One. 2013;8(5):e63378.

157. Liu P, et al. Requirement for Wnt3 in vertebrate axis formation. Nat Genet. 1999;22(4):361-5.

158. Huelsken J, et al. Requirement for beta-catenin in anterior-posterior axis formation in mice. J Cell Biol. 2000;148(3):567-78.

159. Wu J, et al. An alternative pluripotent state confers interspecies chimaeric competency. Nature. 2015;521(7552):316-21.

160. Joo JY, et al. Establishment of a primed pluripotent epiblast stem cell in FGF4-based conditions. Sci Rep. 2014;4:7477.

161. Chou YF, et al. The growth factor environment defines distinct pluripotent ground states in novel blastocyst-derived stem cells. Cell. 2008;135(3):449-61.

162. Chang KH, Li M. Clonal isolation of an intermediate pluripotent stem cell state. Stem Cells. 2013;31(5):918-27.

163. Rathjen J, et al. Formation of a primitive ectoderm like cell population, EPL cells, from ES cells in response to biologically derived factors. J Cell Sci. 1999;112(Pt 5):601-12.

164. Thomson JA, et al. Embryonic stem cell lines derived from human blastocysts. Science. 1998;282(5391):1145-7.

165. Bongso A, et al. Isolation and culture of inner cell mass cells from human blastocysts. Hum Reprod. 1994;9(11):2110-7.
166. Silva SS, et al. X-chromosome inactivation and epigenetic fluidity in human embryonic stem cells. Proc Natl Acad Sci U S A. 2008;105(12):4820-5.

167. Levenstein $M E$, et al. Basic fibroblast growth factor support of human embryonic stem cell self-renewal. Stem Cells. 2006;24(3):568-74.

168. Ludwig TE, et al. Derivation of human embryonic stem cells in defined conditions. Nat Biotechnol. 2006;24(2):185-7.

169. Xu C, et al. Basic fibroblast growth factor supports undifferentiated human embryonic stem cell growth without conditioned medium. Stem Cells. 2005;23(3):315-23.

170. Xu RH, et al. Basic FGF and suppression of BMP signaling sustain undifferentiated proliferation of human ES cells. Nat Methods. 2005;2(3):185-90.

171. O'Leary T, et al. Tracking the progression of the human inner cell mass during embryonic stem cell derivation. Nat Biotechnol. 2012;30(3):278-82.

172. Lengner CJ, et al. Derivation of pre-X inactivation human embryonic stem cells under physiological oxygen concentrations. Cell. 2010;141(5):872-83.

173. Hough SR, et al. Single-cell gene expression profiles define self-renewing, pluripotent, and lineage primed states of human pluripotent stem cells. Stem Cell Reports. 2014;2(6):881-95.

174. Hanna J, et al. Human embryonic stem cells with biological and epigenetic characteristics similar to those of mouse ESCs. Proc Natl Acad Sci U S A. 2010;107(20):9222-7.

175. Gafni $O$, et al. Derivation of novel human ground state naive pluripotent stem cells. Nature. 2013;504(7479):282-6.

176. Theunissen TW, et al. Systematic identification of culture conditions for induction and maintenance of naive human pluripotency. Cell Stem Cell. 2014;15(4):471-87.

177. Ware CB, et al. Derivation of naive human embryonic stem cells. Proc Natl Acad Sci U S A. 2014;111(12):4484-9.

178. Chan YS, et al. Induction of a human pluripotent state with distinct regulatory circuitry that resembles preimplantation epiblast. Cell Stem Cell. 2013;13(6):663-75

179. Duggal, G., et al., Alternative routes to induce naive Pluripotency in human embryonic stem cells. Stem Cells, 2015. 33(9): 2686-2698.

180. Carter $M G$, et al. A primitive growth factor, NME7AB, is sufficient to induce stable naive state human Pluripotency; reprogramming in this novel growth factor confers superior differentiation. Stem Cells. 2016;34(4):847-59.

181. Guo G, et al. Naive Pluripotent stem cells derived directly from isolated cells of the human inner cell mass. Stem Cell Reports. 2016;6(4):437-46.

182. Ware CB, et al. Histone deacetylase inhibition elicits an evolutionarily conserved self-renewal program in embryonic stem cells. Cell Stem Cell. 2009;4(4):359-69.

183. Chen $\mathrm{H}$, et al. Reinforcement of STAT3 activity reprogrammes human embryonic stem cells to naive-like pluripotency. Nat Commun. 2015;6:7095.

184. Takashima $Y$, et al. Resetting transcription factor control circuitry toward ground-state pluripotency in human. Cell. 2014;158(6):1254-69.

185. Amit $\mathrm{M}$, et al. Clonally derived human embryonic stem cell lines maintain pluripotency and proliferative potential for prolonged periods of culture. Dev Biol. 2000;227(2):271-8.

186. Itskovitz-Eldor J, et al. Differentiation of human embryonic stem cells into embryoid bodies compromising the three embryonic germ layers. Mol Med. 2000;6(2):88-95.

187. Reubinoff $\mathrm{BE}$, et al. Embryonic stem cell lines from human blastocysts: somatic differentiation in vitro. Nat Biotechnol. 2000;18(4):399-404.

188. Warrier, S., et al., Direct comparison of distinct naive pluripotent states in human embryonic stem cells. Nat Commun. 2017 Apr 21;8:15055. doi:10.1038/ ncomms15055.

189. Drukker $\mathrm{M}$, et al. Isolation of primitive endoderm, mesoderm, vascular endothelial and trophoblast progenitors from human pluripotent stem cells. Nat Biotechnol. 2012;30(6):531-42.

190. Golos TG, Giakoumopoulos M, Gerami-Naini B. Review: Trophoblast differentiation from human embryonic stem cells. Placenta. 2013; 34(Suppl):S56-61.

191. Xu RH, et al. BMP4 initiates human embryonic stem cell differentiation to trophoblast. Nat Biotechnol. 2002;20(12):1261-4.

192. Li Y, Parast MM. BMP4 regulation of human trophoblast development. Int J Dev Biol. 2014:58(2-4):239-46.

193. Bernardo AS, et al. BRACHYURY and CDX2 mediate BMP-induced differentiation of human and mouse pluripotent stem cells into embryonic and extraembryonic lineages. Cell Stem Cell. 2011;9(2):144-55.

194. James D, et al. Contribution of human embryonic stem cells to mouse blastocysts. Dev Biol. 2006;295(1):90-102. 
195. Masaki $H$, et al. Interspecific in vitro assay for the chimera-forming ability of human pluripotent stem cells. Development. 2015;142(18):3222-30.

196. Nakamura T, et al. A developmental coordinate of pluripotency among mice, monkeys and humans. Nature. 2016:537(7618):57-62.

197. Blakeley $P$, et al. Defining the three cell lineages of the human blastocyst by single-cell RNA-seq. Development. 2015;142(18):3151-65.

198. Huang K, Maruyama T, Fan G. The naive state of human pluripotent stem cells: a synthesis of stem cell and preimplantation embryo transcriptome analyses. Cell Stem Cell. 2014;15(4):410-5.

199. Niakan KK, Eggan K. Analysis of human embryos from zygote to blastocyst reveals distinct gene expression patterns relative to the mouse. Dev Biol. 2013;375(1):54-64

200. Deglincerti $A$, et al. Self-organization of the in vitro attached human embryo. Nature. 2016;533(7602):251-4.

201. Shahbazi MN, et al. Self-organization of the human embryo in the absence of maternal tissues. Nat Cell Biol. 2016;18(6):700-8.

202. Moignard V, et al. Decoding the regulatory network of early blood development from single-cell gene expression measurements. Nat Biotechnol. 2015;33(3):269-+.

203. Scialdone A, et al. Resolving early mesoderm diversification through single-cell expression profiling. Nature. 2016;535(7611):289-+.

204. Wen J, et al. Single-cell analysis reveals lineage segregation in early postimplantation mouse embryos. J Biol Chem. 2017;292:9840-854.

205. Guo GJ, et al. Resolution of cell fate decisions revealed by single-cell Gene expression analysis from zygote to Blastocyst. Dev Cell. 2010;18(4):675-85.

206. Ohnishi Y, et al. Cell-to-cell expression variability followed by signal reinforcement progressively segregates early mouse lineages. Nat Cell Biol. 2014;16(1):27-37.

207. Johansson BM, Wiles MV. Evidence for involvement of activin a and bone morphogenetic protein 4 in mammalian mesoderm and hematopoietic development. Mol Cell Biol. 1995;15(1):141-51.

\section{Submit your next manuscript to BioMed Central and we will help you at every step:}

- We accept pre-submission inquiries

- Our selector tool helps you to find the most relevant journal

- We provide round the clock customer support

- Convenient online submission

- Thorough peer review

- Inclusion in PubMed and all major indexing services

- Maximum visibility for your research

Submit your manuscript at www.biomedcentral.com/submit 